\title{
Article \\ Modal Verification and Strength Analysis of Bladed Rotors of Turbine in Rated Working Conditions
}

\author{
Chang-Sheng Lin ${ }^{1, *} \mathbb{C}$, Hung-Tse Chiang ${ }^{1}$, Chuan-Hsing Hsu ${ }^{1}$, Ming-Hsien Lin ${ }^{1}$, Jui-Kai Liu ${ }^{1}$ and Chi-Jeng Bai ${ }^{2}$ \\ 1 Department of Vehicle Engineering, National Pingtung University of Science and Technology, \\ Pingtung 91201, Taiwan; m10838023@mail.npust.edu.tw (H.-T.C.); m10838027@mail.npust.edu.tw (C.-H.H.); \\ m10838007@mail.npust.edu.tw (M.-H.L.); jkliu@mail.npust.edu.tw (J.-K.L.) \\ 2 Green Energy \& System Integration Research \& Development Department, Technology Division, \\ China Steel Corporation, Kaohsiung 81233, Taiwan; 189654@mail.csc.com.tw \\ * Correspondence: changsheng@mail.npust.edu.tw; Tel.: +886-8-7703202 (ext. 7454)
}

check for updates

Citation: Lin, C.-S.; Chiang, H.-T.; Hsu, C.-H.; Lin, M.-H.; Liu, J.-K.; Bai, C.-J. Modal Verification and Strength Analysis of Bladed Rotors of Turbine in Rated Working Conditions. Appl. Sci. 2021, 11, 6306. https://doi.org/ 10.3390/app11146306

Academic Editor: Paolo Neri

Received: 24 May 2021

Accepted: 30 June 2021

Published: 8 July 2021

Publisher's Note: MDPI stays neutral with regard to jurisdictional claims in published maps and institutional affiliations.

Copyright: (c) 2021 by the authors. Licensee MDPI, Basel, Switzerland. This article is an open access article distributed under the terms and conditions of the Creative Commons Attribution (CC BY) license (https:// creativecommons.org/licenses/by/ $4.0 /)$.

\begin{abstract}
The Top-pressure Recovery Turbine (TRT) uses the blast furnace gas generated in the iron and steel manufacturing process to push the turbine which drives the generator to generate electricity, and the generated electric energy is supplied to in-plant equipment. In this paper, we investigate the aerodynamic force, centrifugal force, and maximum stress on the structure of the TRT rotor in rated working conditions and the positions of occurrence using the Finite Element Method (FEM), as well as discuss the dynamic characteristics of bladed disks during TRT operation through Campbell and SAFE diagrams. To confirm the effectiveness of the finite element models, the mode shapes and natural frequencies in the FEA-based modal analysis of the TRT rotor will be captured and compared with those of the practical structures through the Experimented Modal Analysis (EMA). To verify the agreement between the mode shapes of the finite element analysis and those of the actual structure, the Modal Assurance Criterion (MAC) is introduced here to confirm the reliability of the finite element model. The stress distribution on the structure in the rotation is obtained by centrifugal force analysis. The TRT rotor is driven as the blast furnace top pressure pushes the moving blade; when the rotor rotates, the moving blade bears centrifugal and periodic aerodynamic forces. The stress distribution is investigated on the structure when these forces act simultaneously using aerodynamic analysis. To discuss whether the bladed disks will resonate with the external force under the operating conditions, Campbell and SAFE diagrams are used for evaluation, and the modal parameters obtained from the EMA are used to estimate the strength and durability of the blades. According to the analysis results when the TRT rotor is in working conditions, the fatigue failure may occur at the maximum stress existing on the dovetail slot.
\end{abstract}

Keywords: Top-pressure Recovery Turbine (TRT); bladed disks structures; aerodynamic force; centrifugal force; experimental modal analysis (EMA); finite element analysis (FEA); Campbell diagram; SAFE diagrams

\section{Introduction}

A great amount of energy is consumed in the course of iron and steel manufacture. To recover the waste heat and gas in the production process and to convert them into reusable energy, the TRT is used in the blast furnace for energy recovery [1,2]. The TRT uses the blast furnace gas generated in the operation process of the blast furnace to push the turbine, and the turbine drives the generator to generate electricity. The aerodynamic force is converted into mechanical energy by using the aforesaid method, and the generated electric energy is supplied to in-plant equipment to recover energy.

In an axial turbine, the risk of blade damage is considerable. In addition to reducing the efficiency of the turbine, the failed blade may hit other blades and cause greater losses. Since the TRT system is an axial turbine, it is important to avoid the failure of the blades during the operation of the system. During the operation of the system, in addition to 
pure metal fatigue failure, the damage may also be caused by metal corrosion defects, and failure due to the expansion of defects due to resonance [3]. In practice, the locations where axial turbine blades are prone to failure are the bladed disk blades and the upper edges of the dovetail grooves of the blades. Wei et al. [4] found that a large number of cracks occurred in the root of the blade in the steam turbine, and even the blades that failed due to this. After destructive and non-destructive inspections, it was found that the initial defects of the blades were caused by corrosion, which eventually caused cracks to grow under the influence of various excitations during operation, and finally caused the blades to fail. Poursaeidi et al. [5] found a failed blade in a gas turbine, and the damage location was at the center of the blade. After the modal analysis by the FEM, it is found that the blade has a chance to excite the third mode of the blade under working conditions, which will cause the fatigue failure of the blade. Shevkhlari et al. [6] tested the equivalent working conditions of the blades of the steam turbine. After an equivalent test of 500 working hours, the blades failed at the dovetail slot. The reason is that when the blade is manufactured, the geometry of the dovetail groove of the first section of the blade is different from the design, and the stress concentration occurs there, which eventually leads to the occurrence of fatigue failure. Katinic et al. [7] found blade failure resulted from defects at the dovetail groove of a steam turbine blade. The defects are caused by the corrosion effect of the dovetail groove of the blade. Metal fatigue occurred due to structural vibration during operation. Zhao et al. [8] found signs of fretting wear at the dovetail groove of the failed blade, and, at the operating speed, the second mode of the blade aggravated the crack growth at the wear part, and finally caused structural failure. In a TRT system, blast furnace gas must be received from the blast furnace, and its working conditions are more complicated. Liu et al. [9] discussed the causes of TRT blade failure in their research and found that fretting and corrosion occurred at the dovetail groove of the blade at the same time. Finally, under structural vibration, cracks grew until the blade failed.

The centrifugal force generated during the operation of the rotor is also one of the keys to the damage of the blade structure. Cano et al. [10] used the FEM to analyze the cenrtifugal force of blade members, and the assessment of corresponding blades service life can then be carried out. The results of the study show that the centrifugal force during the operation of the structure contributes to the generation of cracks on the blades, and more closely leads to the growth of cracks. The fatigue failure of turbine blades is gradual. The initial blade defects cause cracks to grow gradually, causing blade failure during the process. Rodriguee et al. [11] used a vibration exciter to analyze the natural frequency of a cracked blade and found that when the blade has a crack, the natural frequency of the structure will change, and the position of the crack will change, and the blade vibration amplitude will also change. The longer the crack length, the greater the drop in the natural frequency of the structure. In addition to using vibration exciters for operating modal analysis of the blade structure, the EMA is also a common method to explore the dynamic characteristics of the structure. Zhang et al. [12] used experimental modal analysis to explore the dynamic characteristics of Kaplan Turbine blades, and compared them with the frequency results of the Theoretical Modal Analysis (TMA) based on FEM to improve the reliability of the finite element model. $\mathrm{Wu}$ [13] used experimental modal analysis methods to explore the dynamic characteristics of large turbines. After knowing the dynamic behavior of the system, using the disturbance behavior of the jet airflow to the blades, the frequency that may be excited by the external force is established, and is helpful to the discussion of blade failure analysis.

From previous studies, it can be known that the failure behavior of blades is mostly caused by initial defects. Under the external excitation of the structure, cracks are generated at the defects, and the cracks grow gradually. Therefore, understanding the structural dynamic characteristics of the blade is an important point that cannot be ignored in the rotor structure. The Campbell diagram is a method used to evaluate the dynamic characteristics of the axial flow turbine blade disc structure. The Campbell diagram can effectively determine whether the structure will resonate at the working speed. To 
explore the structural mode of the system resonance, Singh et al. [14] proposed a Singh's Advanced Frequency Evaluation (SAFE) diagram, which integrates the structural modes and resonance frequency information to determine the structural modes that may be excited. Bertini et al. [15] used Campbell diagrams and SAFE diagrams to discuss the dynamic characteristics of the impeller. The results indicated that for the conditions under which the structural mode is excited, except for the same natural frequencies of the structure, the excitation phase of the external force needs to be consistent with the structural modes; otherwise, the structure will not resonate with the excitation. To monitor the moving blades of the turbine effectively, some rotor systems will be equipped with a Blade Tip Timing (BTT) system. The BTT technology can be used to measure the displacement of the tip of the rotor blade, and then analyze the dynamic characteristics of the rotor blade during operation. Madhavan et al. [16] used the BTT system to monitor the blade dynamics and found abnormal blade vibration during the experiment. After shutting down the inspection, they found that the blade heel was cracked, which indicated that the BTT system was effective in monitoring the dynamic characteristics of the blade.

In the past studies, more attention was paid to the dynamic characteristics of the blades, and the simplified equivalent blisk/blade model was used for discussion. In this paper, a more complete model verified by EMA is used to implement strength analysis of the bladed disks of the turbine in rated working conditions. We firstly performed EMA on the practical TRT rotor, and then used modal verification technology to verify and update the finite element model. The centrifugal force analysis is employed to explore the centrifugal force that the TRT rotor structure bears during operation, evaluate the strength of the structure, and verify the stress generated by the centrifugal force at the blade and the analytical solution. In addition to the dynamic characteristics of the blade itself, we also pay attention to the contact behavior at the dovetail groove of the blade. After the verification is completed, the aerodynamic force of the TRT rotor structure is taken into consideration to evaluate the stress of the structure under actual working conditions. In the end, we used Campbell and SAFE diagrams to explore the dynamic characteristics of the TRT bladed disks to estimate the mode shapes of the TRT rotor that may be excited under working conditions.

\section{Materials and Methods}

The analysis of this study is the steelworks of a two-stage dry-type TRT comprising the stationary blades of two stages and moving blades of two stages. When the blast furnace top pressure gas flows into the TRT system, it passes by the 1st stage stationary blades with variable guide vanes at first. The purpose is to control the flow velocity of gas by adjusting the guide vanes to adjust the generating power of the TRT system. After passing by the 1st stage stationary blades, the air flows by the 1st stage moving blade and generates a lift to push the TRT rotor. When the air passes by the 1st stage blades, the flow velocity and pressure decrease to make the 2nd stage moving blade generate the torque equal to that of the 1st stage blade. The air flows by the 2nd stage stationary blades at first to increase the flow velocity. As the gas pressure decreases, to achieve the torque identical to that of the 1st stage, the 2nd stage rotor needs a larger flow area to reduce energy loss. Therefore, the 2nd stage moving blade is longer than the 1st stage moving blade.

This study aims to analyze the strength of the TRT moving blade in working conditions. To improve the reliability of the finite element model of moving blades, we perform modal verification to check the consistency of the actual structure and the corresponding finite element model. We use the MAC to evaluate the correlation between the mode shapes obtained from Theoretical and Experimental modal analysis to check the similarity of the mode shapes. It is difficult to perform in-situ measurements for TRT under working conditions; therefore, we use Computer-Aided-Engineering (CAE) software for analyzing centrifugal force and an aerodynamic force to discuss the stress distribution on the structure when the TRT rotor is in operation condition, and to evaluate the probable initial region if the structure fails. 


\subsection{Modal Verification of TRT Blades}

To investigate the dynamic characteristics of TRT rotor blades, we used commercial software ANSYS to perform modal analysis, centrifugal force analysis, and aerodynamic analysis of TRT rotors through finite element analysis. To improve the reliability of the finite element models, the mode shapes and natural frequencies in the modal analysis of the TRT rotor will be captured and compared with those of the practical structures. To verify the agreement between the mode shapes of the finite element analysis and those of the actual structure, the MAC is introduced here to confirm the reliability of the finite element model.

\subsubsection{Theoretical Modal Analysis of TRT Rotors Based on FEM}

To implement modal verification, we used commercial software ANSYS to create a finite element model of the TRT rotor, which is employed in conjunction with appropriate boundary conditions and load conditions for computer-aided engineering. For the theoretical modal analysis of the TRT rotor, the free-free boundary condition is set, and an appropriate impulse force is applied at the chosen excitation point. Its purpose is to simulate the response of each measurement point of the TRT rotor system subjected to a broadband excitation, and the natural frequencies and mode shapes of the structure can then be obtained from the modal analysis based on finite element method.

\subsubsection{Experimental Modal Analysis of TRT Rotors}

To obtain the modal parameters of the TRT bladed disk structures, an impact hammer is employed to give a pulse excitation to the TRT rotor, the purpose of which is to excite the dynamic characteristics of the TRT blades. We set up an accelerometer at the measuring point, and use the accelerometer to collect the response data generated by the excitation of the TRT rotor blade. After obtaining the excitation and response signals, the excitation and response data are incorporated into the Brüel \& Kjær RT Pro Photon 7.0 data acquisition system, and the Fast Fourier Transform (FFT) of correlation function operations are performed, and then the Frequency Response Function (FRF) of the system is obtained. The experimental procedure is as shown in Figure 1.

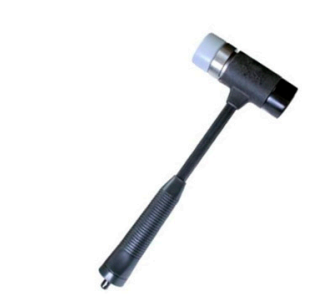

Impact hammer

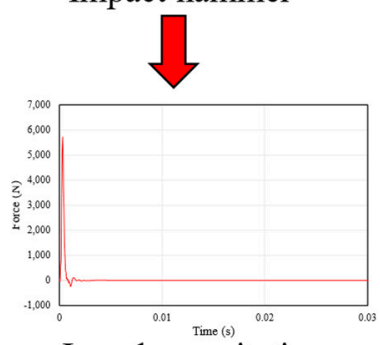

Impulse excitation

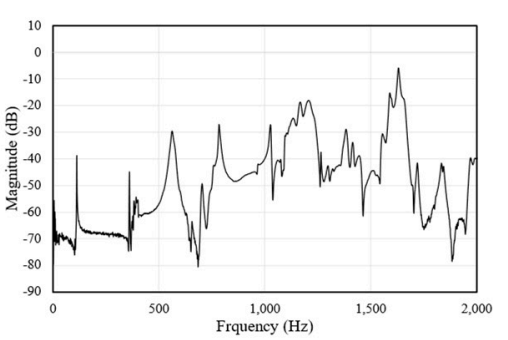

Frequency Response Function

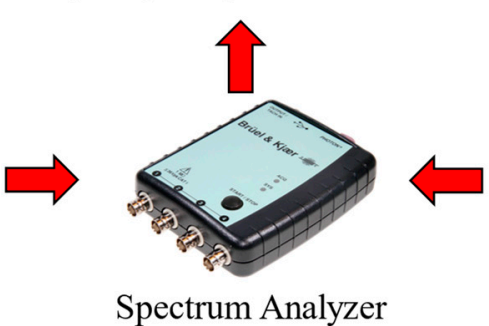

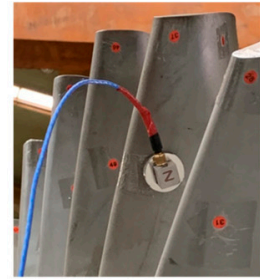

Accelerometer

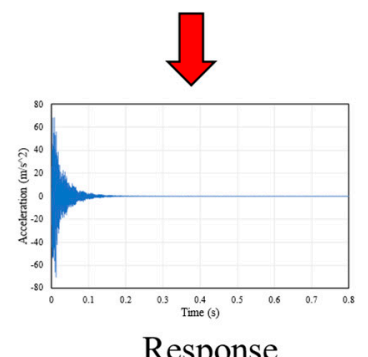

Response

Figure 1. A flowchart of experimental signal analysis.

The response of the system contains the information of excitation and the dynamic characteristics of the system. When the excitation frequency is close to the natural frequency of the system, the system has the largest magnification, which is regarded as resonance. However, the inner product of the mode shape and the excitation vectors is 
equally important to the contribution of resonance, and its physical meaning represents the similarity between the excitation phase (distribution in space) and the phase of the mode shape. When the two phases are similar (or the same), the inner product is larger up to unity; when it is completely orthogonal, the inner product is zero [12]. Therefore, to excite the structural modes with mutually orthogonal direction components effectively, we apply excitations in the axial and radial directions, respectively, to excite the structural modes with axial or radial direction components effectively. In the experiment, since the signal measurement is often susceptible to environmental vibration or contaminated with other noise, it is impossible to guarantee whether the measured response is all induced from the excitation, so the coherence function $\gamma^{2}(f)$ is employed as the importance index for a combination of the FFT of correlation functions between the response and excitation data, which is defined as

$$
\gamma^{2}(f)=\frac{\left|G_{f x}(f)\right|^{2}}{G_{x x}(f) G_{f f}(f)}
$$

where $G_{x x}(f), G_{f f}(f)$, and $G_{f x}(f)$ are, respectively, the auto power spectral density function of the system responses, the auto power spectral density function of the input excitation, and the cross-power spectral density function between the response and the excitation. $\gamma^{2}(f)$ is close to unity, which means that the input and output data have a fairly good correlation. On the contrary, the closer the value is to zero, it means that the correlation between input and output data is not good. After evaluating $\gamma^{2}(f)$ to estimate the correlation between the response and the excitation data, we evaluate the frequency response function (FRF) $H_{x f}(f)$ of the system by using the combination of the power spectrum density function $G_{f f}(f)$ and $G_{f x}(f)$ defined as follows:

$$
H_{x f}(f)=\frac{G_{f x}(f)}{G_{f f}(f)}
$$

The FRF combined with the auto- and cross-power spectrum used in this study is one of the frequency-domain modal identifications of the structure under ambient vibration. This method is based on the assumption that the input signal is stationary white noise under the condition of unknown excitation, and the characteristics of the correlation function are employed to estimate the modal parameters of the system by evaluating the auto-power spectrum of the structural response data and the magnitude and phase of the cross-power spectrum with the corresponding response data of a reference channel.

\subsubsection{Modal Assurance Criterion (MAC)}

To ensure the consistency of the mode shapes obtained from the theoretical modal analysis and the experimental modal analysis, we use the modal assurance criteria (MAC) proposed by Allemang [17] and defined as follows:

$$
\operatorname{MAC}\left(\varphi_{i A}, \varphi_{j X}\right)=\frac{\left|\left\{\varphi_{i A}\right\}^{T}\left\{\varphi_{i X}\right\}^{*}\right|^{2}}{\left\{\varphi_{i A}\right\}^{T}\left\{\varphi_{i A}\right\}^{*}\left\{\varphi_{i X}\right\}^{T}\left\{\varphi_{i X}\right\}^{*}}
$$

where $\varphi_{i A}$ and $\varphi_{j X}$ are the mode-shape vectors obtained from theoretical modal analysis and experimental modal analysis, respectively. $T$ and $*$ are, respectively, transpose and conjugate complex operators of the vector or matrix. Using the $M A C$ indicator can verify the agreement between the mode-shape vectors obtained from finite element analysis and experimental modal analysis. If the $M A C$ value approaches zero, it means that the correlation between the two modes is low; on the contrary, if the $M A C$ is close to unity, it means that the correlation between the two modes is high. 


\subsection{Analysis of Centrifugal Force of TRT Rotor}

When the TRT rotor is in operation, it must bear the centrifugal force resulting from rotation. The centrifugal force will occur in the radial direction of the rotating shaft, so that the blade bears the tensile stress resulted from centrifugal force during rotation, as shown in Figure 2.

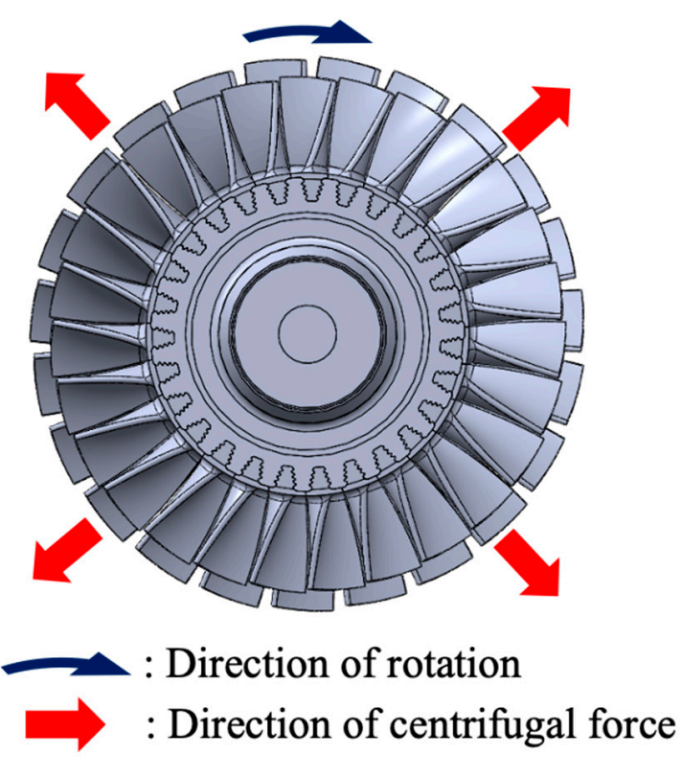

Figure 2. Centrifugal force resulting from rotor blades of TRT rotor in operation.

To discuss the influence of centrifugal force on the TRT rotor blade, two techniques are used for analysis and validation. First, the analytic solution is used for the determination of tensile stress of blade, and the tensile stress caused from centrifugal force of blade is analyzed by using the FEM. The analysis results are compared with the analytic solution, and then verified the reliability of centrifugal force analysis.

\subsubsection{Analytical Solution}

The TRT rotor working speed is $3600 \mathrm{rpm}$ (60 rps). To obtain the tensile stress distribution on the blade due to centrifugal force, the centrifugal force $\left(F_{r}\right)$ shall be calculated which is expressed as follows:

$$
F_{r}=m \omega^{2} l
$$

where $m$ is the object mass; $\omega$ is the angular velocity of an object rotating along the shaft; $l$ is the shortest distance between the object centroid and rotation axis. According to the above equation, the magnitude of centrifugal force is correlated with the rotational speed of the object and related to the mass and centroid position of the object. As the length direction of the blade is normal to the direction of centrifugal force, there are different centrifugal forces in different length positions of the blade, expressed as follows:

$$
F_{r}=\int_{r-R_{H u b}}^{R_{T i p}-R_{H u b}} \rho A_{\kappa} \omega^{2} D_{r} d \kappa
$$

where $R_{\text {Tip }}$ is the distance from the center of circle $\mathbf{O}$ to the blade tip; $R_{H u b}$ is the distance from the center of circle $\mathbf{O}$ to the blade root; $r$ is the distance from the center of circle $\mathbf{O}$ to the blade section of the desired centrifugal force; $\rho$ is the blade material density; $\omega$ is the angular velocity of rotation of the rotor; $A_{\kappa}$ is the function of the locational relation between the cross-section area and blade length; $D_{r}$ is the function of the locational relation between the centroid position and blade length. The aforesaid symbols and blade diagram are shown in Figure 3. The centrifugal force on the section of arbitrary blade length positions can be obtained through the aforesaid procedure. 


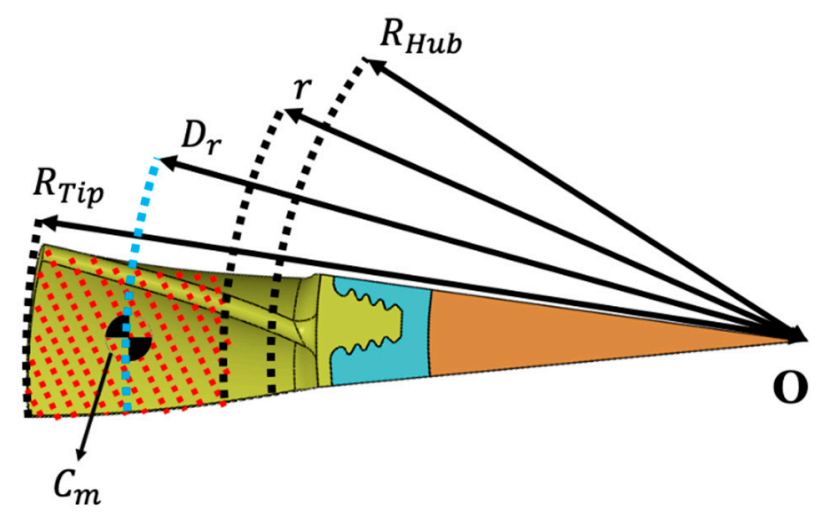

Figure 3. Schematic diagram of a part of rotor blades.

When the centrifugal force in each length position of the blade is obtained, the tensile stress $\left(\sigma_{r}\right)$ in various positions of the blade can be calculated which is expressed as follows:

$$
\sigma_{r}=\frac{F_{r}}{A_{r}}
$$

where $\sigma_{r}$ is the tensile stress resulting from centrifugal force; $F_{r}$ is the centrifugal force; and $A_{r}$ is the cross-sectional area. The tensile stress resulting from the centrifugal force in any radius position of the blade can be obtained by Equations (4)-(6).

\subsubsection{Centrifugal Force Analysis of TRT Rotor Based on FEM}

To discuss the stress intensity resulting from the centrifugal force in various positions of the TRT rotor blade, the CAE software is used for analyzing the centrifugal force of the TRT rotor. In the centrifugal force analysis, the load and boundary conditions are set up according to actual working conditions. The shaft speed is set as $3600 \mathrm{rpm}$, and the axial and radial constraints are given where the shaft bearing is fixed. After analysis, the stress of each section is extracted and compared with the analytic solution; the reliability of analysis is enhanced; and the influence of maximum stress on the structure is evaluated.

\subsection{Analysis of Aerodynamic Force of TRT Rotor}

The TRT system uses blast furnace top pressure to push the rotor blade, and the shaft drives the generator to generate electricity. When the rotor is in operation, the blast furnace gas flows across the flow channel between stationary blades, and the blast furnace gas is transferred to the moving blade. In this working condition, the moving blade moves among different flow channels due to the rotation of the shaft, so the external force on the moving blade is a periodic function. In actuation conditions, the aerodynamic force on the moving blade is a periodic function, and the aerodynamic load change can be expressed as follows.

$$
F_{A}=F_{A 0}+F_{A 1} \sin \left(\omega_{b} t+\theta_{1}\right)+F_{A 2} \sin \left(\omega_{b} t+\theta_{2}\right)+\ldots+F_{A m} \sin \left(\omega_{b} t+\theta_{m}\right)
$$

where $F_{A}$ is the external force on the moving blade; $F_{A 0} \sim F_{A m}$ are the magnitude of aerodynamic force flowing through the flow channel between different stationary blades when the rotor is in operation; $\omega_{b}$ is the frequency of the moving blade passing through the flow channel when the rotor is in operation; and $\theta_{1} \sim \theta_{m}$ are the phase difference of aerodynamic force between flow channels. According to Equation (7), the aerodynamic external force of various moving blades in different time conditions can be calculated as long as the magnitude of the aerodynamic force, the frequency of the rotor blade passing through the flow channel, and the phase difference of aerodynamic force between flow channels can be obtained.

The phase difference of aerodynamic force between flow channels has resulted from unequal numbers of moving blades and flow channels of the TRT rotor. Therefore, the 
phase difference is related to the geometry of the moving blade and flow channel. The law of phase difference repetition can be obtained by calculating the greatest common factor between the number of moving blades and the number of stationary blades, and the phase difference is calculated in the minimum unit. The minimum phase difference $\theta_{\min }$ is calculated as the following equation:

$$
\theta_{\min }=\frac{360}{N_{s}} \times \frac{g c f\left(N_{d}, N_{s}\right)}{N_{d}}
$$

where $N_{d}$ is the number of moving blades; $N_{s}$ is the number of stationary blades; $\operatorname{gcf}\left(N_{d}, N_{s}\right)$ is the greatest common factor of moving blades and the number of stationary blades. After the angle of minimum phase difference and the law of phase difference repetition are obtained, the phase difference of each blade can be calculated by the following equation.

$$
\theta_{m}=\theta_{\min } \times N_{o}
$$

where $\theta_{m}$ is the aerodynamic phase difference of the m-th moving blade; $\theta_{\min }$ is the minimum phase difference; $N_{o}$ is the repetition number of the phase difference. To obtain the ordinal number between the blade and flow channel, the following equation is used for computation.

$$
\begin{gathered}
B_{m}=\bmod \left(m, \frac{\left[g c f\left(N_{d}, N_{s}\right)\right]}{N_{d}}\right) \\
B_{m}=0, N_{o}=\frac{N_{d}}{g c f\left(N_{d}, N_{s}\right)}-1 \\
B_{m}>0, N_{o}=B_{m}-1
\end{gathered}
$$

Equation (11) uses the geometrical relationship between the moving blade and flow channel to calculate the ordinal number between the moving blade and flow channel. $B_{m}$ is the parameter of discriminant; $m$ is the desired blade number; $\operatorname{gcf}\left(N_{d}, N_{s}\right)$ is the greatest common factor of the moving blade and the number of stationary blades; $N_{d}$ is the number of moving blades; mod is the remainder operator; the calculated constituent ordinal number is substituted in Equation (9) to obtain the aerodynamic phase difference of moving blade $\mathrm{m}$.

In the aerodynamic load change (Equation (7), besides the phase difference of aerodynamic force between flow channels $\left(\theta_{1} \sim \theta_{m}\right)$, the frequency $\omega_{b}$ of the moving blade passing through the flow channel when the rotor is in operation must be known. The frequency of moving blades passing through the flow channel can be obtained through the following procedure.

$$
\omega_{b}=\frac{M N}{60}
$$

where $\omega_{b}$ is the frequency of the blade passing by the nozzle when the rotor is in operation; $M$ is the number of flow channels of stationary blades on the circumference; and $N$ is the working speed. The calculation results of Equations (9) and (12) are substituted in Equation (4) to obtain the aerodynamic force parameter on the moving blade.

The finite element aerodynamic analysis is used for discussing the stress behavior of the shaft and blade of the TRT rotor under the effect of the aerodynamic force in working conditions. In the analysis, the loading conditions are set up according to actual working conditions; a simple harmonic external force is applied to the face of each blade as per the result of Equation (4). Besides loading conditions, the boundary condition setting for the TRT rotor in practice is of great importance, given the boundaries' aim to simulate the constraints of the TRT rotor in the actual structure. The main referential boundary is the interface of the connection between the bearing on the rotor and the generator.

\subsection{Campbell and SAFE Diagrams of TRT Bladed Disk}

When the TRT rotor is under operation, the frequency in rotation obtained from order analysis will be generated due to the movement of the shaft. The frequency in 
rotation obtained from order analysis will vary with the number of blades. When the order frequency is close to the natural frequency of the structure, the mode shape of the structure system will be excited, causing the structure to vibrate. To understand the possibility that the rotor is excited by the frequency in rotation obtained from order analysis when the rotor is running, the theoretical modal analysis is employed to explore the dynamic behavior of the rotor at different speeds, and we can then obtain the Campbell diagram containing the relationship between the modal frequency and speed of the system at different speeds. The purpose of the Campbell diagram is to evaluate whether the shaft and blades will be excited by the frequency in rotation obtained from order analysis under the working speed of the system, which will cause the vibration of the structural system.

The Campbell diagram can be used to explore the relationship between the natural frequencies of the TRT rotor and the frequency in rotation obtained from order analysis, but it is unavailable to obtain the mode shapes of the excited structural modes. To understand the information of mode shapes at the intersection of the order line and the modal frequency of structural systems further, the SAFE diagram is applied to explore the types of nodal diameters to corresponding structural modes by drawing the structural modes with different nodal diameters of all moving blades at different speeds. The information contained in the SAFE diagram includes the system frequency where resonance occurs and the number of nodal diameters of its mode shapes; SAFE-diagram-related information can be used to estimate the durability of the blades further.

\section{Results and Discussion}

\subsection{Modal Verification of TRT Rotor}

To explore whether the finite element model is equivalent to the actual structure, we use experimental modal analysis to extract the modal parameters of the actual structure and compare them with the modal parameters obtained from the theoretical modal analysis. In addition to the error comparison of the natural frequencies, the MAC is also used to compare the mode shapes to improve the reliability of the geometric model.

\subsubsection{Theoretical Modal Analysis of TRT Rotor}

The finite element model of the TRT rotor is established in commercial software. The finite element type and its parameters are shown in Figure 4 and Table 1. After completing the establishment of the finite element model, the material of the model of the TRT rotor is chosen as SUS630, and the corresponding material parameters are shown in Table 2. In order to obtain the mode shapes of the TRT rotor, the boundary condition of the TRT rotor is set as a free-free condition, and an impulse excitation is applied to the TRT rotor to obtain the natural frequencies and mode shapes of the structural system. Through the Theoretical modal analysis, all the mode shapes in the bandwidth of interest can be obtained, and the position of the measurement point of the blades can be selected based on mode shapes of a structure.

Table 1. Information of finite element model.

\begin{tabular}{cc}
\hline \multicolumn{2}{c}{ Finite Element Model Information } \\
\hline Numbers of nodes of finite element model & $1,500,225$ \\
\hline Numbers of elements of finite element model & $1,565,132$ \\
\hline Element type & solid hexahedral \\
\hline
\end{tabular}




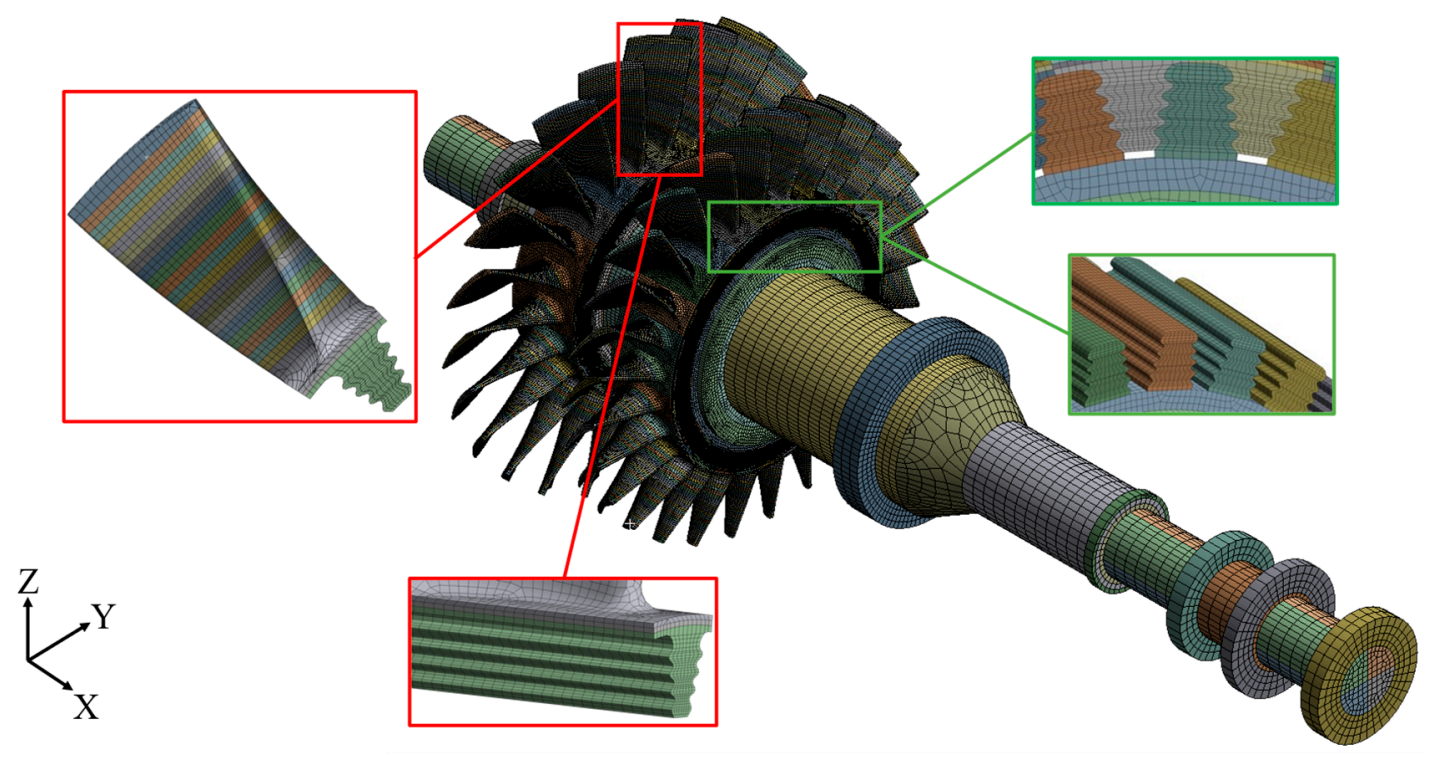

Figure 4. Finite element model of TRT rotor.

Table 2. Material parameters of TRT rotor.

\begin{tabular}{cc}
\hline & Mechanical Property \\
\hline Material type & SUS630 \\
\hline Density $\left(\mathrm{kg} / \mathrm{m}^{3}\right)$ & 7750 \\
\hline Young's modulus $(\mathrm{GPa})$ & 197 \\
\hline Poisson ratio & 0.27 \\
\hline Yield stress (MPa) & 724 \\
\hline Ultimate stress (MPa) & 930 \\
\hline
\end{tabular}

The blade is a curved structure, so the coordinate direction of each degree of freedom of measurement in the experiment is different to define, and the global coordinate system (global coordinate system) cannot be used for data acquisition. To make the coordinates set in the experimental modal analysis consistent with those in finite element analysis, we redefine a local coordinate system for each degree of freedom of measurement on the blade in the finite element model, as shown in Figure 5, and the analysis result can then be represented in the local coordinate system. Note also that in Figure 5, the red lines are the $X_{n}$-axis directions of the local coordinate systems, which are the definition of the $Y_{n^{-}}$ and $\mathrm{Zn}$ - directions in Cartesian coordinates; the green lines are the $Y_{n}$-axis directions of the local coordinate systems, which are defined along the tangent direction of the blade surfaces; the blue lines are the $\mathrm{Zn}$-axis directions of the local coordinate systems, defined along the normal directions of the blade surfaces.

According to the theoretical modal analysis, the mode shapes of the TRT rotor mainly belong to the bending modes of the shaft, and its typical mode shape is shown in Figure 6. The mode shapes of the blades belonging to the combination of the bending and torsion modes are shown in Figure 7. 


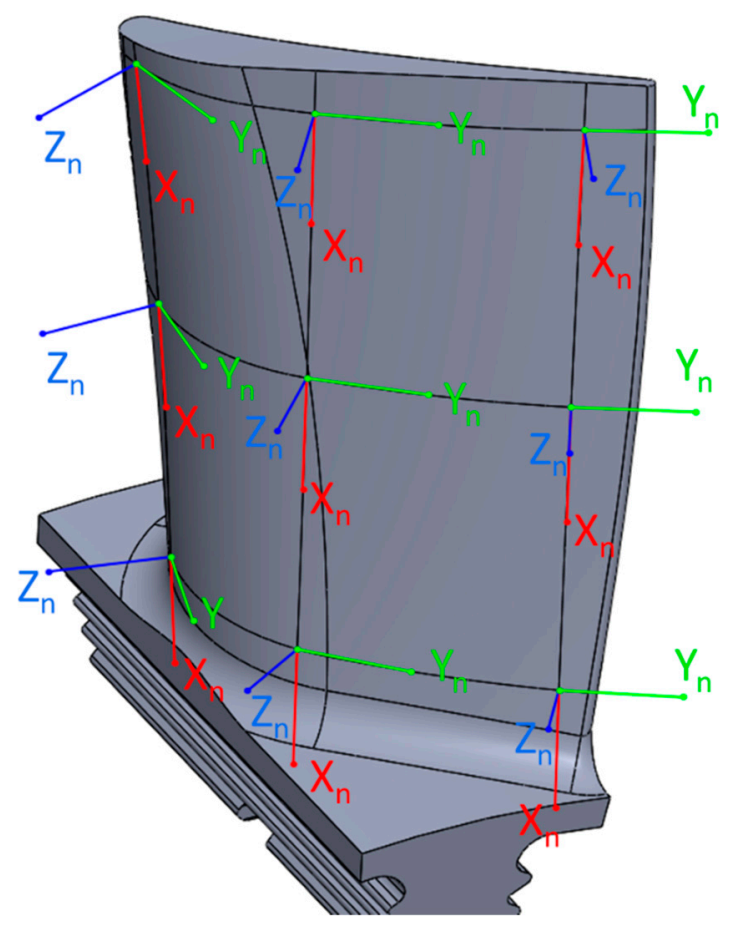

Figure 5. Schematic diagram of measuring point on the blade in the local coordinate systems.

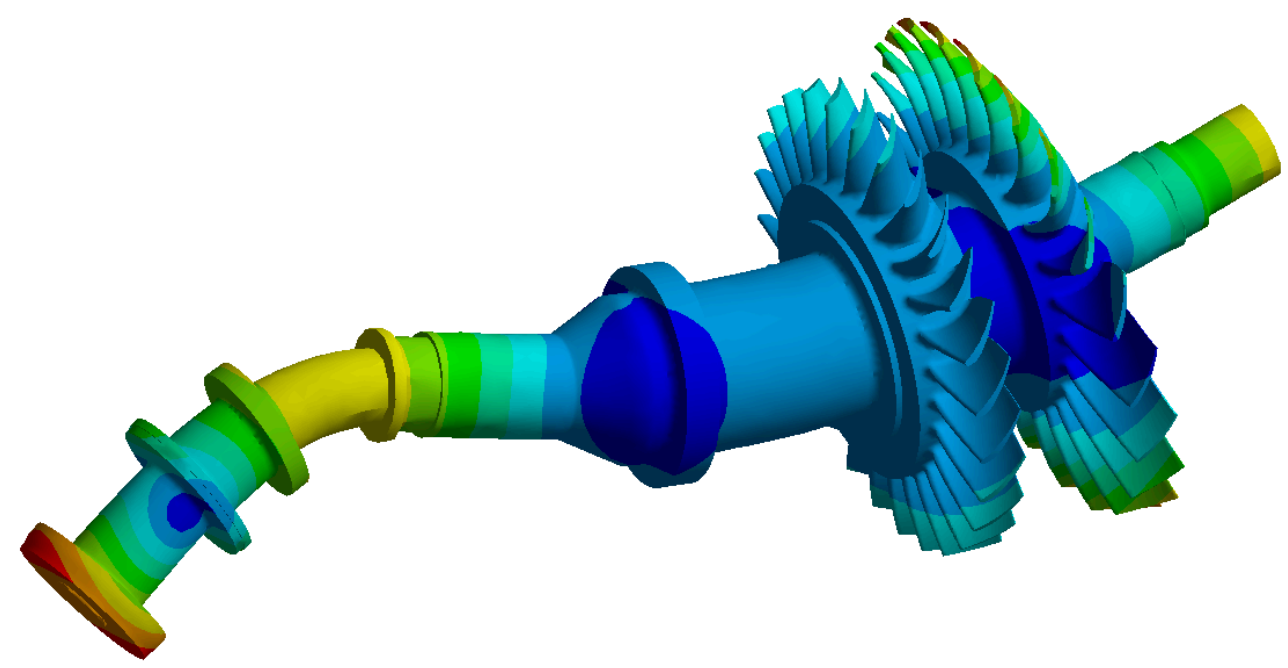

Figure 6. A typical mode shape of TRT rotor.
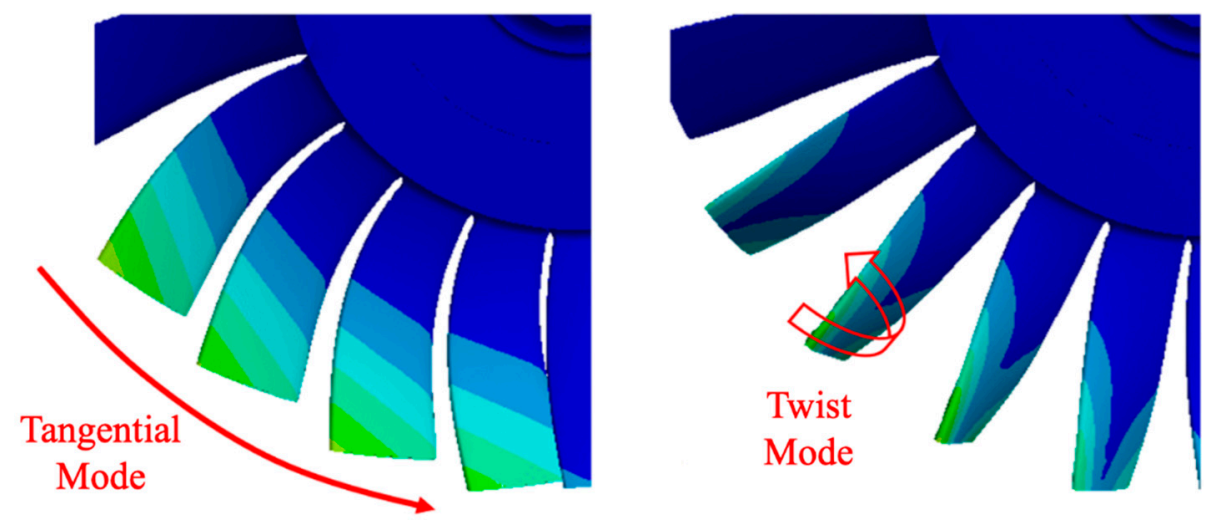

Figure 7. A typical mode shape of TRT blades. 


\subsubsection{A Set Up of Modal Testing of TRT Rotor}

In the experimental modal analysis, the X-direction excitation is carried out to excite the mode shapes of the TRT rotor completely. In the experiment, the excitation is applied to the clock position of twelve o'clock, which means directly ahead, on the disc surface of the 2nd stage bladed disk, and the excitation direction is the $\mathrm{X}$ direction (axial direction). The schematic diagram of the experiment and the test status in modal testing are shown in Figures 8 and 9.

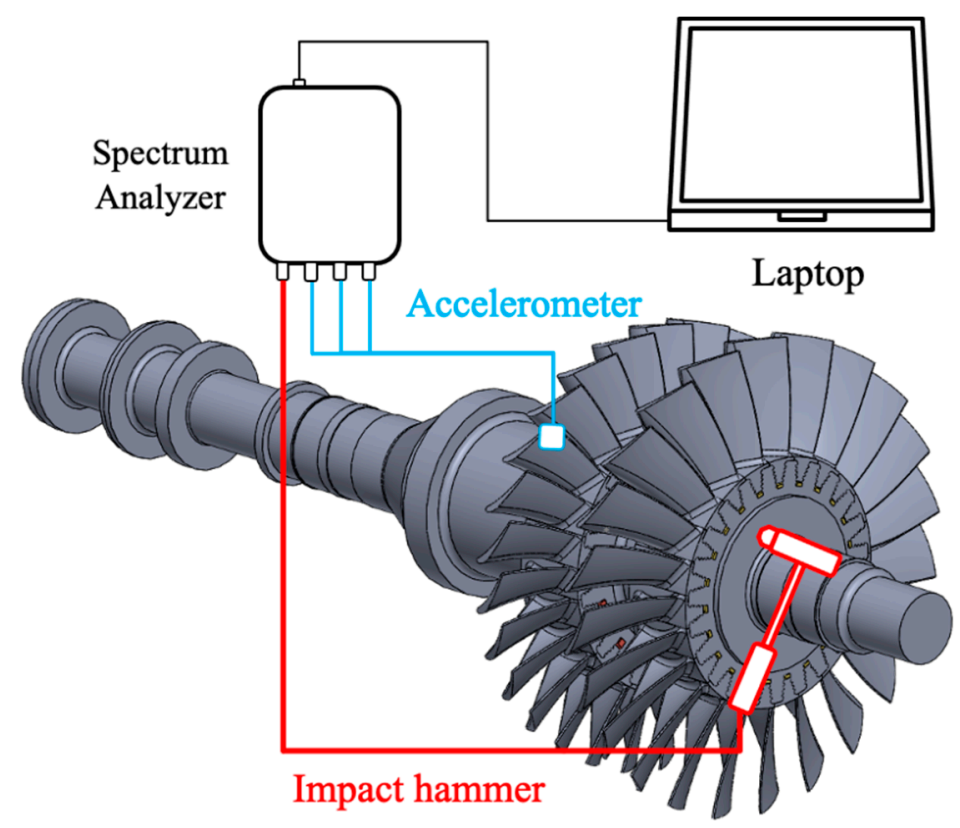

Figure 8. Schematic diagram of $X$ direction and the clock position of twelve o'clock meaning directly ahead of the excitation applied in modal testing.

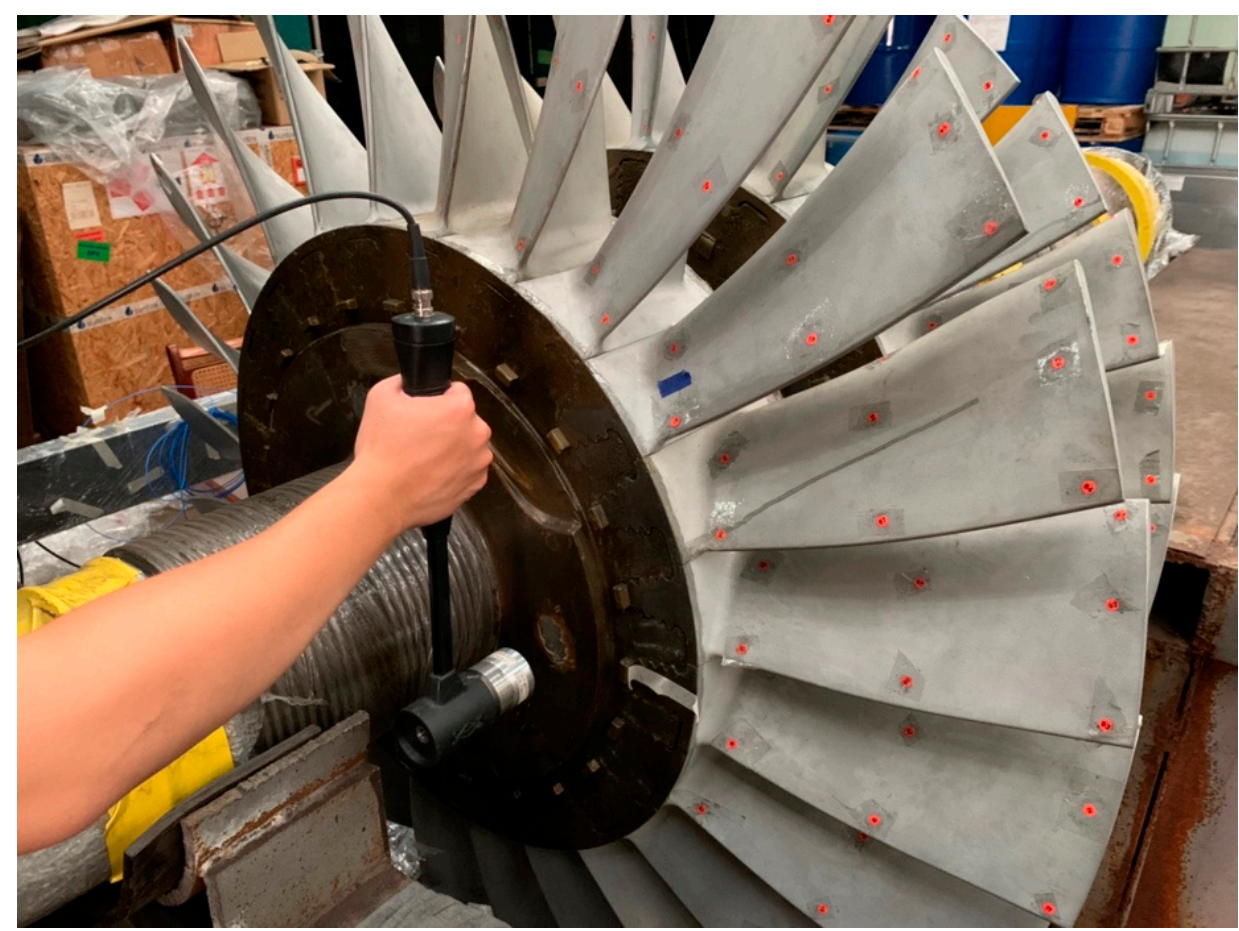

Figure 9. A typical $X$ direction and the clock position of three o'clock meaning directly to the right of the excitation applied in modal testing of in-situ measurements. 
To obtain the modal information on the blades fully, each blade is arranged with nine degrees of freedoms. To be able to arrange accurately that the degree of freedom of measurement is in the position to be observed on the actual structure, we use six plastic ropes to position and mark the positioning lines, and install the marked plastic ropes on the TRT rotor blades, and then quickly locate the position of the degree of freedom of measurement. This skill not only effectively reduces the difference in measurement positions in between modal testing and the finite element model, but also can effectively carry out the arrangement procedure of measurement points before performing modal testing. The arrangement of the degree of freedom of measurement is shown in Figure 10.

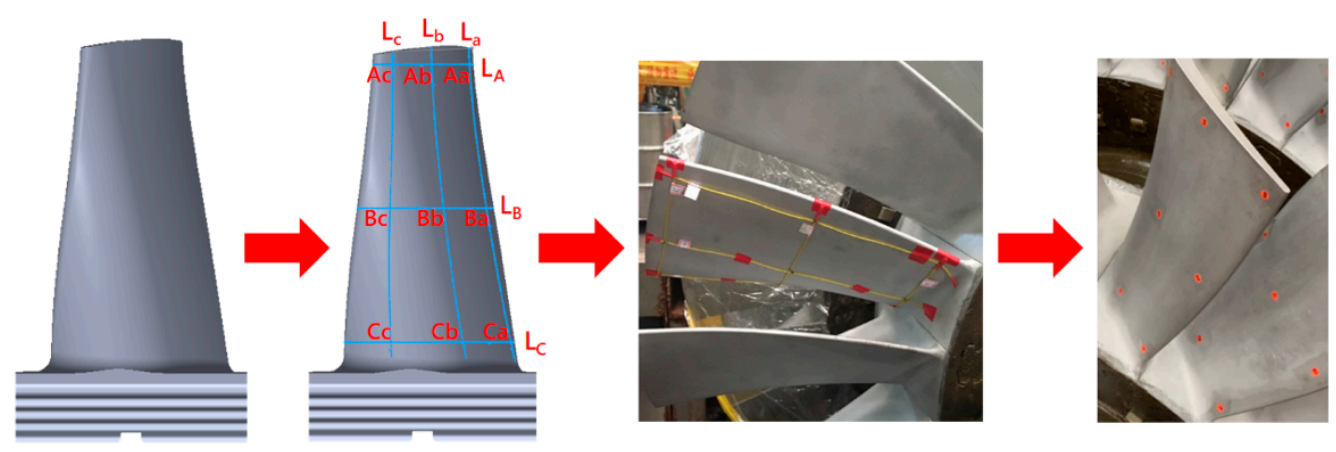

Figure 10. The arranged measurement points of blade in modal testing.

In this study, the bandwidth of interest is $1-2000 \mathrm{~Hz}$. To ensure that the signal within the bandwidth to be observed does not produce aliasing effects, based on the Nyquist Theorem, the sampling bandwidth should be set to twice the highest frequency. Since frequency overlap may still occur in the frequency bandwidth above $80 \%$, the sampling bandwidth should be set to 2.5 times or more. Based on the capability of computer calculations, the sampling bandwidth should be set to 2.56 times or more. It can be seen that if the frequency of a signal to be observed is within $2000 \mathrm{~Hz}$, the sampling bandwidth should be set to $5120 \mathrm{~Hz}$ or more. Due to measurement limitations of the frequency range of the Brüel \& Kjær RT Pro Photon 7.0 data acquisition system, the sampling bandwidth of this experiment is set to $8000 \mathrm{~Hz}$; the number of samples is 6400; and the signal sampling time is $1.25 \mathrm{~s}$.

\subsubsection{Experimental Modal Analysis of TRT Rotor}

The test system planned the excitation position at the clock position of twelve o'clock meaning directly ahead on the disk surface of the 2nd stage bladed disk, and tried to excite the mode shapes of the TRT rotor in the X direction (axial direction) effectively. After obtaining the frequency response function of each measuring point of the moving blade through the percussion test, we imported the frequency response function of each degree of freedom of measurement into the ME' Scope commercial software, and extracted the natural frequencies and mode shapes of the structure by $\mathrm{ME}^{\prime}$ Scope. To explore whether the mode shapes obtained from the finite element analysis and the experimental modal analysis are consistent, the modal assurance criterion is introduced here to estimate the agreement between mode shapes. The comparison results of the natural frequencies and the mode shapes are shown in Table 3. 
Table 3. Results of modal frequencies of the first and 2nd stage bladed disk.

\begin{tabular}{|c|c|c|c|c|c|c|c|}
\hline \multicolumn{4}{|c|}{ The 1st Stage Bladed Disk } & \multicolumn{4}{|c|}{ The 2nd Stage Bladed Disk } \\
\hline $\begin{array}{l}\text { FEA } \\
(\mathrm{Hz})\end{array}$ & $\begin{array}{l}\text { EMA } \\
(\mathbf{H z})\end{array}$ & Error (\%) & MAC & $\begin{array}{l}\text { FEA } \\
(\mathrm{Hz})\end{array}$ & $\begin{array}{c}\text { EMA } \\
\text { (Hz) }\end{array}$ & Error (\%) & MAC \\
\hline 110.10 & 112.20 & 1.90 & 0.98 & 110.10 & 112.20 & 1.90 & 0.94 \\
\hline 334.80 & 361.40 & 7.90 & 0.79 & 334.80 & 361.60 & 8.00 & 0.50 \\
\hline 428.10 & 378.90 & 11.50 & 0.70 & 523.60 & 562.30 & 7.40 & 0.83 \\
\hline 425.20 & 392.10 & 7.80 & 0.65 & 674.70 & 701.80 & 4.00 & 0.60 \\
\hline 523.60 & 701.50 & 34.00 & 0.49 & 760.00 & 782.80 & 3.00 & 0.40 \\
\hline 674.60 & 707.60 & 4.90 & 0.53 & 1121.80 & 1024.50 & 8.70 & 0.52 \\
\hline 755.90 & 740.50 & 2.00 & 0.54 & 1367.30 & 1367.20 & 0.00 & 0.80 \\
\hline 766.80 & 750.70 & 2.10 & 0.60 & 1462.80 & 1385.30 & 5.30 & 0.50 \\
\hline 812.00 & 761.00 & 6.30 & 0.72 & 1463.50 & 1396.70 & 4.60 & 0.52 \\
\hline 931.80 & 969.4 & 4.00 & 0.58 & 1482.60 & 1413.00 & 4.70 & 0.48 \\
\hline 1017.00 & 1025.20 & 0.80 & 0.64 & 1516.60 & 1452.20 & 4.20 & 0.74 \\
\hline 1120.70 & 1097.10 & 2.10 & 0.42 & 1583.70 & 1478.80 & 6.60 & 0.59 \\
\hline 1462.80 & 1379.20 & 5.70 & 0.63 & 1698.40 & 1635.40 & 3.70 & 0.93 \\
\hline 1463.50 & 1392.90 & 4.80 & 0.43 & 1901.40 & 1787.80 & 6.00 & 0.89 \\
\hline 1580.50 & 1563.40 & 1.10 & 0.45 & 1973.00 & 1839.10 & 6.80 & 0.62 \\
\hline 1707.70 & 1628.50 & 4.60 & 0.54 & 1983.10 & 1845.40 & 6.90 & 0.87 \\
\hline 1978.00 & 1839.90 & 7.00 & 0.71 & & & & \\
\hline 1980.80 & 1845.60 & 6.80 & 0.71 & & & & \\
\hline 1982.50 & 1972.40 & 0.50 & 0.62 & & & & \\
\hline 2002.60 & 2051.30 & 2.40 & 0.61 & & & & \\
\hline
\end{tabular}

\subsection{Analysis of Centrifugal Force of TRT Rotor}

In centrifugal force analysis, the stress on the blade and shaft and the stress distribution positions when the TRT rotor is in a working condition of $3600 \mathrm{rpm}$ will be discussed in the following.

\subsubsection{Analytic Solution Calculation Results}

The stress intensity in various length positions of the blade can be calculated by Equations (5) and (6); the stress on each section of the blade is shown in Figure 11.

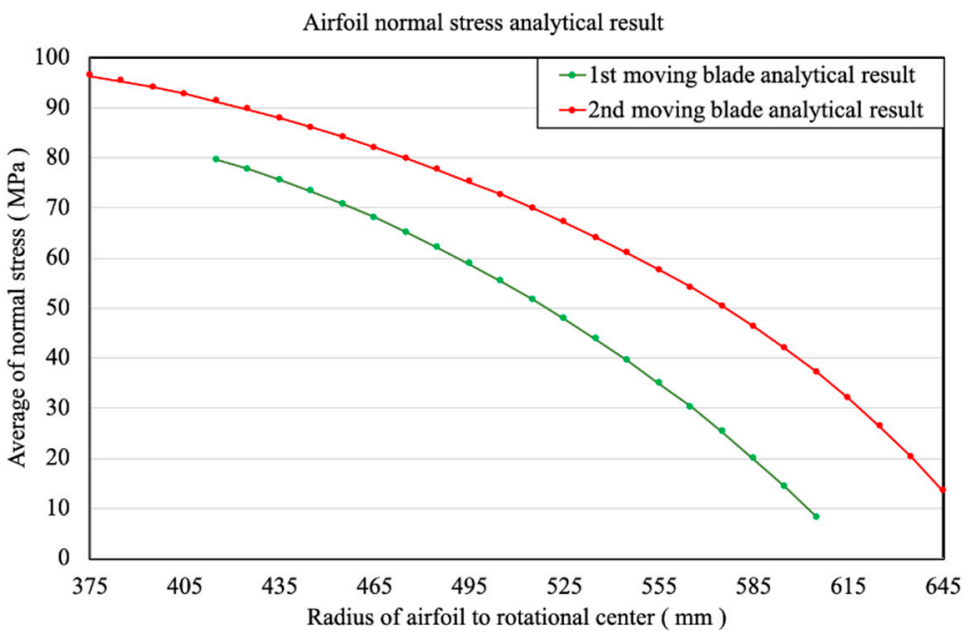

Figure 11. Analytic solution calculation results of the 1st stage and 2nd stage centrifugal stresses.

According to the analytic solution of the 1st stage and 2nd stage blades, the maximum stress of the 1st stage blade is $79.60 \mathrm{MPa}$, and the maximum stress of the 2nd stage blade is $96.30 \mathrm{MPa}$, which occurs at the bottom of the blade. The stress, induced by centrifugal force, decreases towards the blade tip because the cross-section area closer to the blade tip receives less centrifugal force pulled by local mass. 


\subsubsection{Centrifugal Force Analysis Results}

The stress intensity on the wingspan of the blade when the blade works at $3600 \mathrm{rpm}$ is calculated in the analytic solution result. To explore the contact stress on the TRT moving blade further, the computer-aided analysis software is used for centrifugal force analysis. To make the analysis condition match the actual situation, axial and radial constraints are applied to the bearing, and the rotational degree of freedom of the shaft is maintained. The contact pair is set between the blade and shaft in the dovetail slot; the purpose is to know the contact stress intensity on the dovetail slot when the blade is pulled by centrifugal force in centrifugal action. When the shaft speed is $3600 \mathrm{rpm}$, the stress on the TRT rotor is shown in Figure 12.
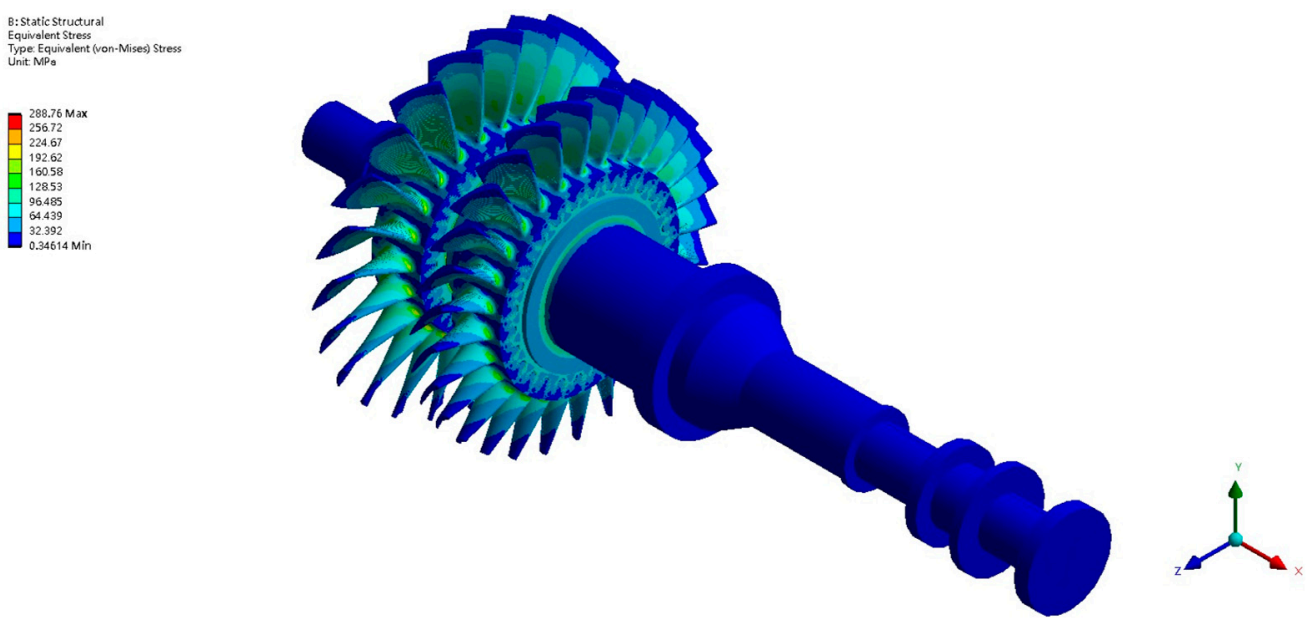

Figure 12. Stress result of TRT rotor centrifugal force analysis.

The average stresses on various sections of the 1st stage and 2nd stage moving blades in the simulation are extracted, and the simulation result is compared with the analytic solution. The two results have coincident trends and almost identical intensity, so the finite element model is reliable. The two stresses are compared in Figure 13.

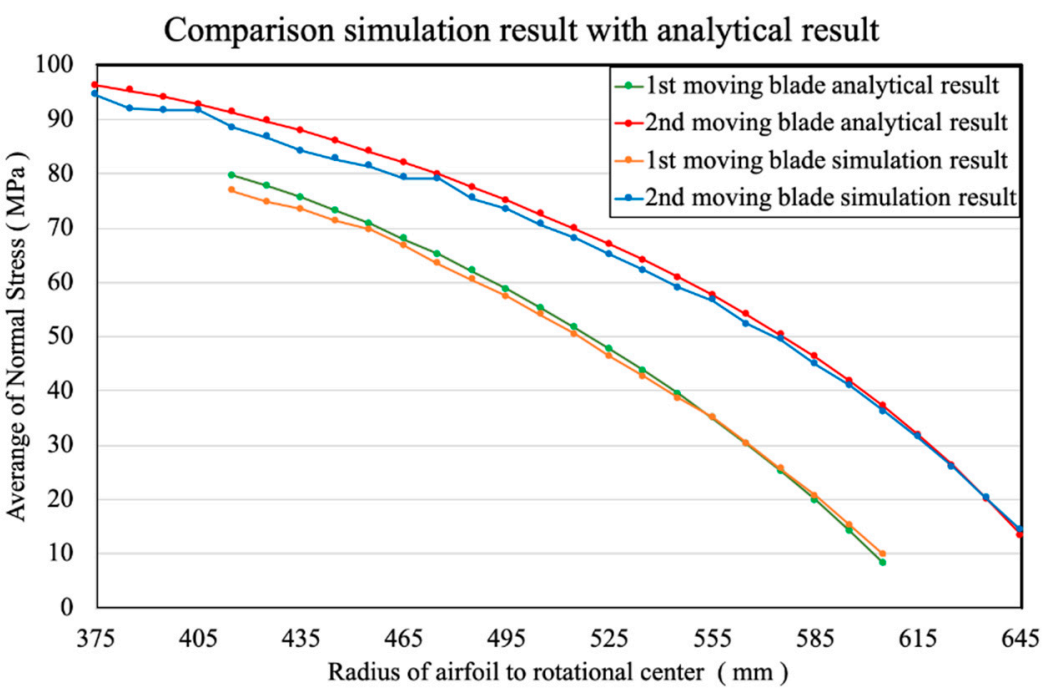

Figure 13. Comparison diagram of analytic solution and simulation of centrifugal stress on TRT blade.

To estimate the difference between the stress results of the analytic solution and analysis, the errors in the two results are calculated, respectively. The mean error of the 1 st stage moving blade is $0.20 \%$; the root mean squared error of the 1st stage moving blade is $4.98 \%$. The error mean of the 2 nd stage moving blade is $2.12 \%$; the root mean squared 
error of the 2nd stage moving blade is $2.90 \%$. The errors in the simulation and analytic solution are less than $5 \%$, so the analysis result is reasonable.

When the coincidence of the simulation calculation result and the analytic solution is confirmed, the maximum stress on the shaft is not in the wingspan of the blade, but in the first section dovetail slot of the blade. As shown in Figure 14, when the 1st stage moving blade of the TRT rotor is extracted for discussion, it is found that the maximum stress of the 1st stage moving blade occurs in the first section dovetail slot; the stress value is $259.21 \mathrm{MPa}$.
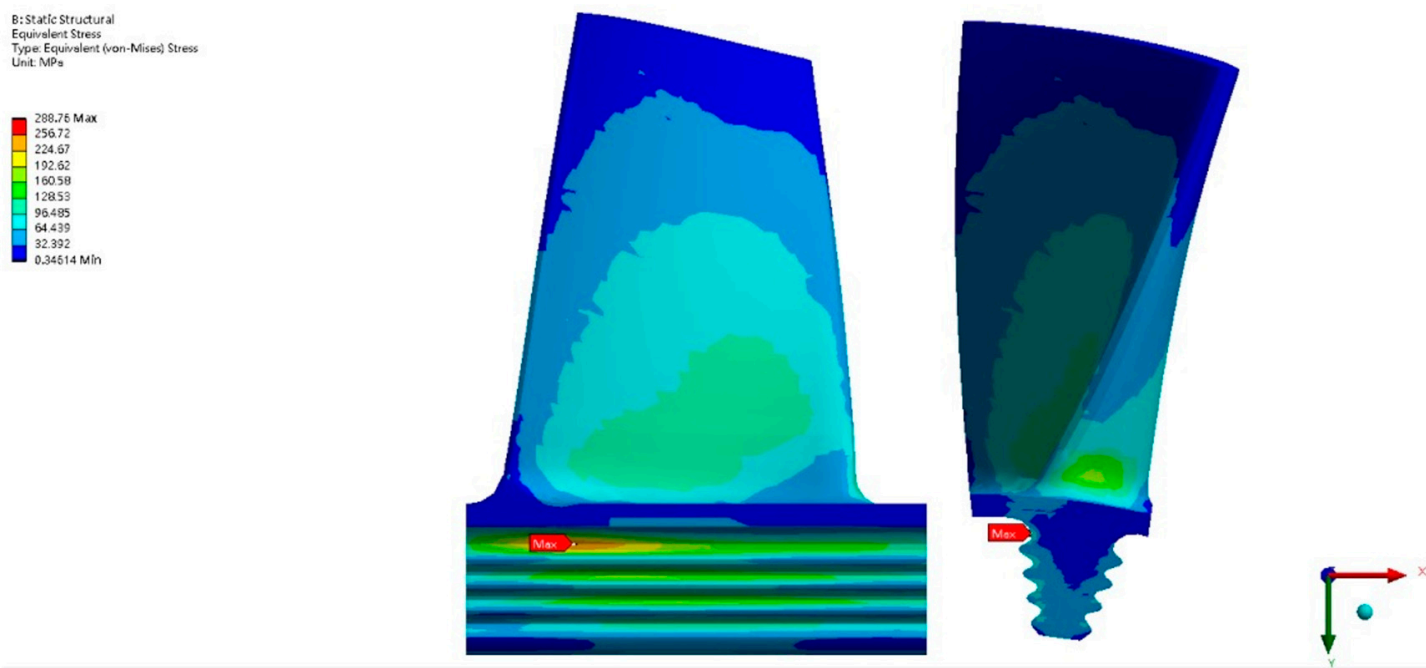

Figure 14. Stress result of centrifugal force analysis of the 1st stage moving blade.

As shown in Figure 15, the maximum stress occurs in the first section dovetail slot of the 2nd stage blade. The maximum stress of the 2nd stage moving blade is $286.21 \mathrm{MPa}$; it is the region of the TRT rotor bearing the maximum stress.
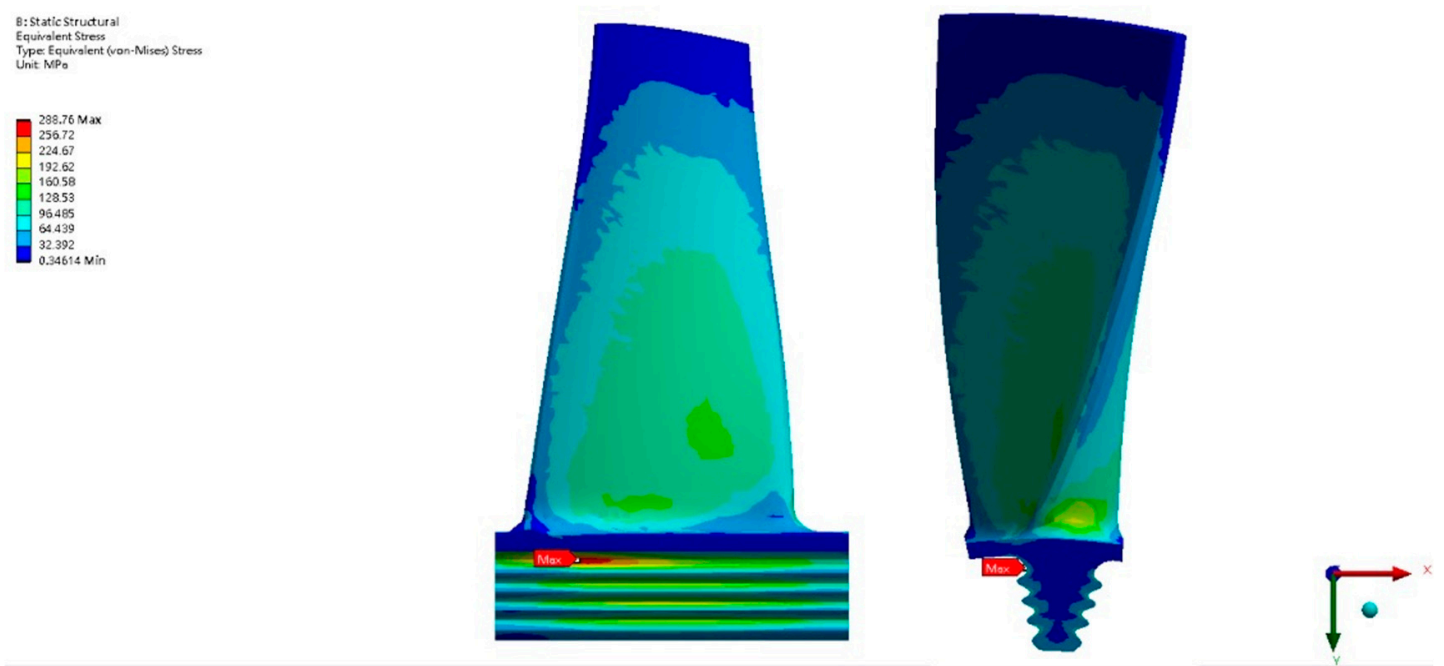

Figure 15. Stress result of centrifugal force analysis of the 2nd stage moving blade.

According to the overall centrifugal force analysis result, the maximum stress occurs in the inner side of the dovetail slot of the 2nd stage blade; the stress value is $286.21 \mathrm{MPa}$. The maximum stress is lower than the yield stress $(735 \mathrm{MPa})$, so the structure will not have plastic deformation under the effect of centrifugal force. 


\subsection{Analysis of Aerodynamic Force of TRT Rotor}

In the aerodynamic analysis, the stress resulting from the centrifugal force of rotation and the aerodynamic force through the flow channel and the stress distribution positions when the TRT rotor is actuated will be discussed.

To investigate the stress behavior of the TRT moving blade in working conditions resulting from the centrifugal force of rotation and the aerodynamic force of the driving system, the FEM is used for analyzing centrifugal and aerodynamic forces. To make the analysis condition match the actual situation, axial and radial constraints are applied to the bearing, and the rotational degree of freedom of the shaft is maintained. The contact pair is set between the blade and shaft in the dovetail slot aiming to determine the contact stress intensity on the dovetail slot when the blade receives a centrifugal force in centrifugal action.

To analyze the maximum air pressure on the windward side of the moving blade when the TRT rotor system is in operational condition, the operating load parameter of the moving blade must be obtained at first. According to the data provided by steelworks, the generating power of the TRT system is correlated with the blast furnace inlet air pressure and the variable guide vanes opening. The result is shown in Figure 16.
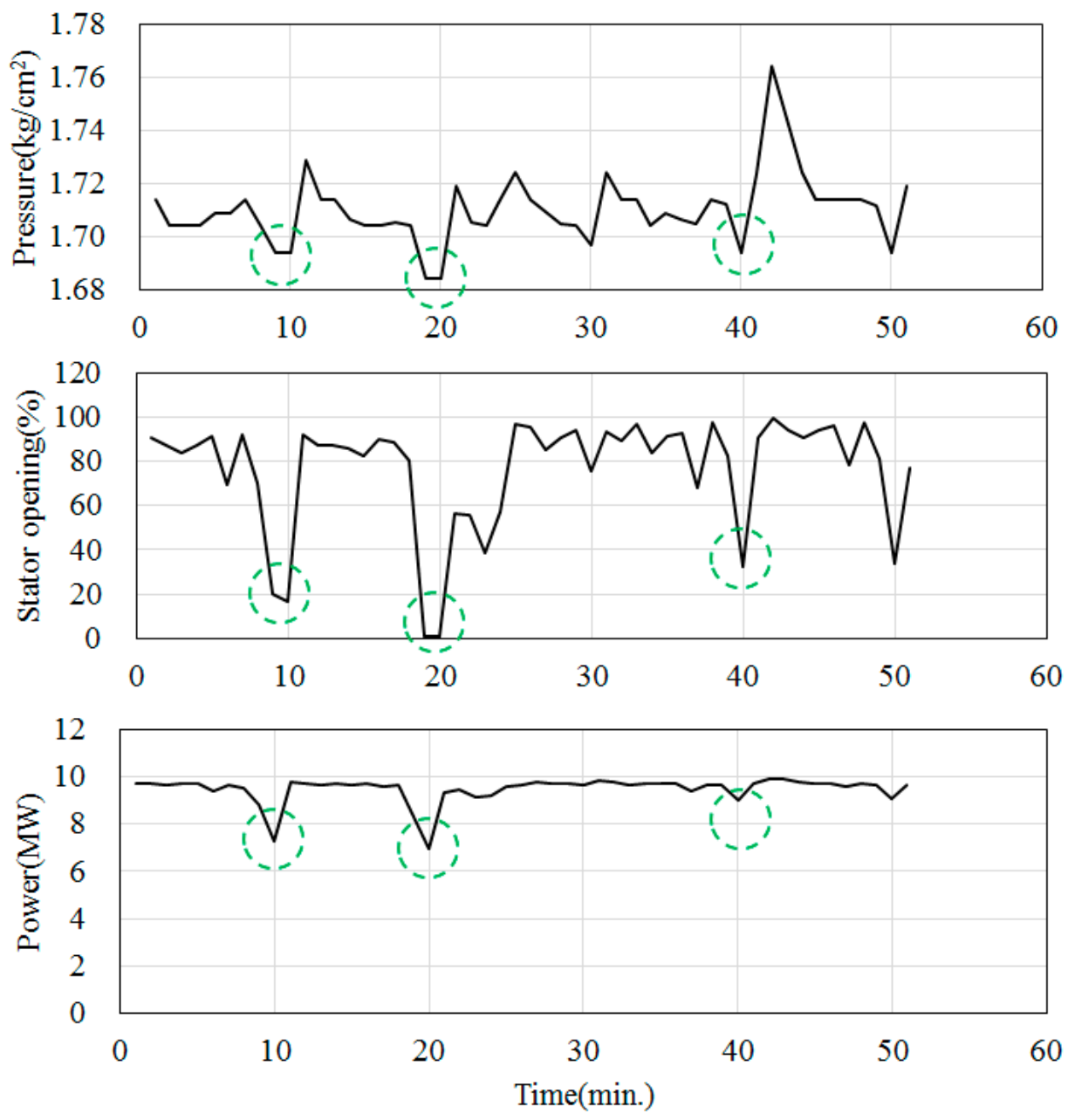

Figure 16. Operation conditions of inlet pressure, stationary blades' opening, and power.

The CFD analysis result of the TRT flow field provided by steelworks shows the pressure on the 1st stage and 2nd stage moving blade faces, and the maximum pressure on the blade face is used as the loading condition of aerodynamic analysis. The TRT flow field analysis result is shown in Table 4. 
Table 4. CFD analysis result.

\begin{tabular}{cc}
\hline \multicolumn{2}{c}{ Working Condition Parameter } \\
\hline Inlet pressure $(\mathrm{kPa})$ & 173.58 \\
Outlet pressure $(\mathrm{kPa})$ & 11.77 \\
Stationary blades opening $(\%)$ & 100.00 \\
Torque of 1st moving blade $(\mathrm{N}-\mathrm{m})$ & 538.08 \\
Torque of 2nd moving blade $(\mathrm{N}-\mathrm{m})$ & 815.62 \\
Rotation shaft work $(\mathrm{MW})$ & 12.55 \\
Maximum pressure on 1st moving blade $(\mathrm{kPa})$ & 213.30 \\
Maximum pressure on 2nd moving blade $(\mathrm{kPa})$ & 155.62 \\
Average pressure on 1st moving blade $(\mathrm{kPa})$ & 105.59 \\
Average pressure on 1st moving blade $(\mathrm{kPa})$ & 377.60 \\
\hline
\end{tabular}

When the aerodynamic force parameter of actuation is obtained, the maximum load when the TRT rotor blade is in operation condition can become known. However, when the TRT rotor is in operation, the moving blade cuts the flow fields of various flow channels, so when each blade bears aerodynamic force, it does not bear a constant external force but bears a periodic simple harmonic external force. The working condition of this system is a constant speed of $3600 \mathrm{rpm}$, so the periodic function of the aerodynamic force of the moving blade is $\cos (60 \times$ number of stationary blades). As the number of stationary blades is unequal to the number of moving blades, the aerodynamic force on the moving blade will have a phase difference. The law of phase difference repetition can be known by calculating the greatest common factor between the number of moving blades and the number of stationary blades. The phase difference of aerodynamic force between flow channels is calculated by Equations (7) and (8). The calculation results are shown in Figures 17 and 18, and Table 5.

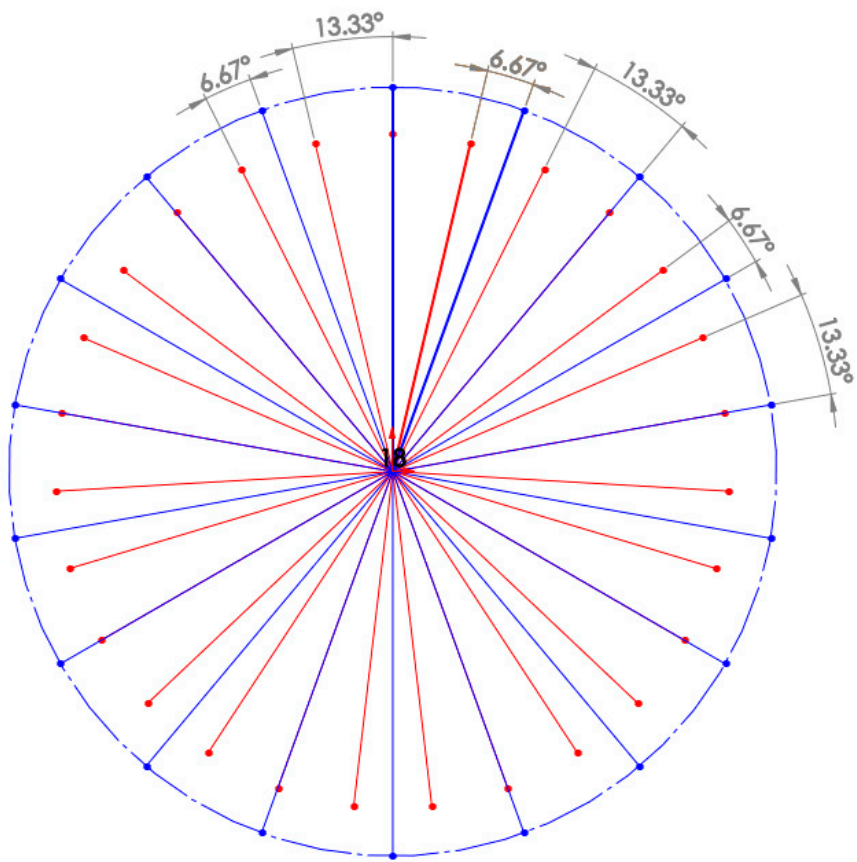

Figure 17. The phase difference of the 1st stage stationary blade and moving blade. 


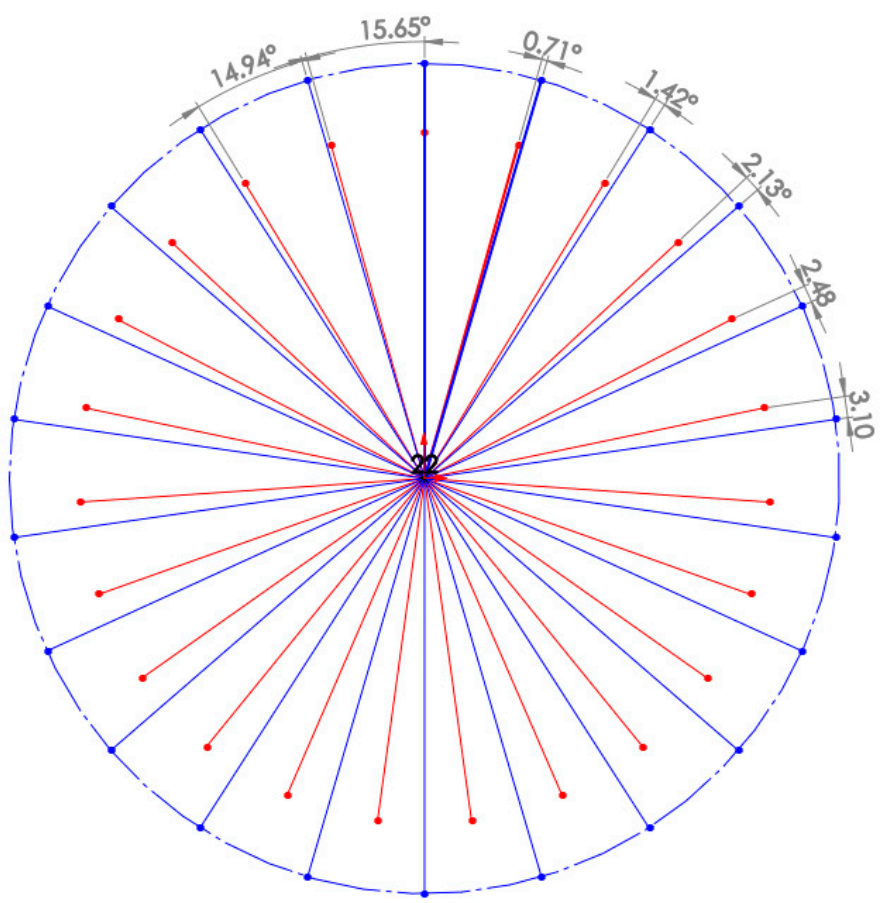

Figure 18. The phase difference of the 2nd stage moving blade.

Table 5. The phase difference of aerodynamic action of moving blade.

\begin{tabular}{|c|c|c|c|}
\hline $\begin{array}{c}\text { 1st Stage Moving } \\
\text { Blade (\#) }\end{array}$ & $\begin{array}{l}\text { Phase Angle } \\
\text { (Degree) }\end{array}$ & $\begin{array}{c}\text { 2nd Stage Moving } \\
\text { Blade (\#) }\end{array}$ & $\begin{array}{c}\text { Phase Angle } \\
\text { (Degree) }\end{array}$ \\
\hline 1 & 0 & 1 & 0 \\
\hline 2 & 6.67 & 2 & 0.71 \\
\hline 3 & 13.33 & 3 & 1.42 \\
\hline 4 & 0 & 4 & 2.13 \\
\hline 5 & 6.67 & 5 & 2.84 \\
\hline 6 & 13.33 & 6 & 3.55 \\
\hline 7 & 0 & 7 & 4.26 \\
\hline 8 & 6.67 & 8 & 4.97 \\
\hline 9 & 13.33 & 9 & 5.68 \\
\hline 10 & 0 & 10 & 6.39 \\
\hline 11 & 6.67 & 11 & 7.10 \\
\hline 12 & 13.33 & 12 & 7.81 \\
\hline 13 & 0 & 13 & 8.52 \\
\hline 14 & 6.67 & 14 & 9.23 \\
\hline 15 & 13.33 & 15 & 9.94 \\
\hline 16 & 0 & 16 & 10.65 \\
\hline 17 & 6.67 & 17 & 11.36 \\
\hline 18 & 13.33 & 18 & 12.07 \\
\hline 19 & 0 & 19 & 12.78 \\
\hline 20 & 6.67 & 20 & 13.49 \\
\hline 21 & 13.33 & 21 & 14.20 \\
\hline 22 & 0 & 22 & 14.91 \\
\hline 23 & 6.67 & 23 & 15.62 \\
\hline 24 & 13.33 & - & - \\
\hline 25 & 0 & - & - \\
\hline 26 & 6.67 & - & - \\
\hline 27 & 13.33 & - & - \\
\hline
\end{tabular}

According to the CFD analysis result of the TRT flow field provided by steelworks (Table 4), when the inlet pressure is $1.77 \mathrm{~kg} / \mathrm{cm}^{2}$ and the stationary blades' opening is $100 \%$, the harshest working condition of the TRT moving blade is formed. Therefore, the 
maximum pressure on the 1st stage and 2nd stage moving blades in this condition is set as the aerodynamic condition of the rotor blade. The air loads of two blades (amplitude of simple harmonic external force) are 1st stage blade $0.21 \mathrm{MPa}$, 2nd stage blade $0.16 \mathrm{MPa}$.

When the amplitude of simple harmonic external force and the phase difference of aerodynamic force in various flow channels are obtained, the parameters are substituted in Equation (7) to obtain the function of simple harmonic external force on each moving blade. After the external force result is obtained, each external force is applied to the windward side of each moving blade, as shown in Figure 19.
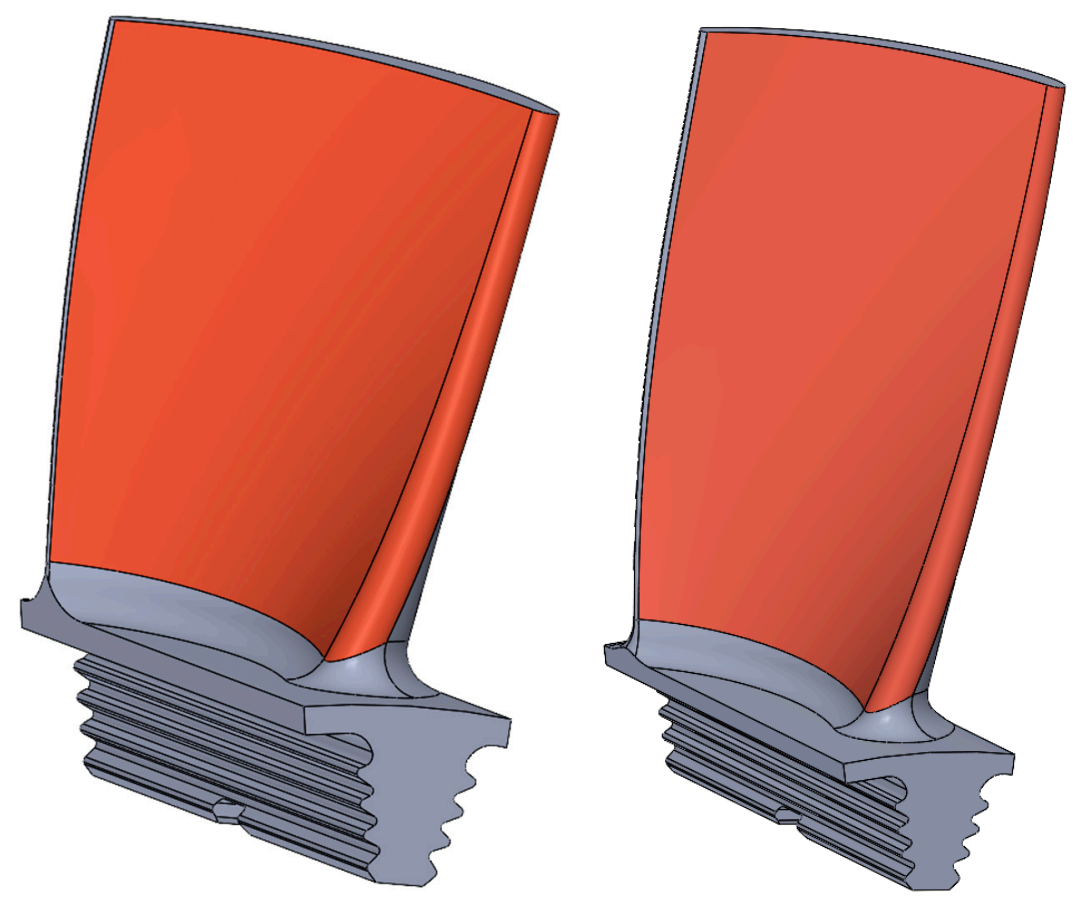

Figure 19. Upper and lower surface positions.

In this analysis, to examine the composition effect of aerodynamic force and centrifugal force, besides the aerodynamic force resulting from the flow field, the structure is given a $3600 \mathrm{rpm}$ rotation effect in the aerodynamic analysis. The detailed loading and boundary conditions are illustrated below in Figure 20.

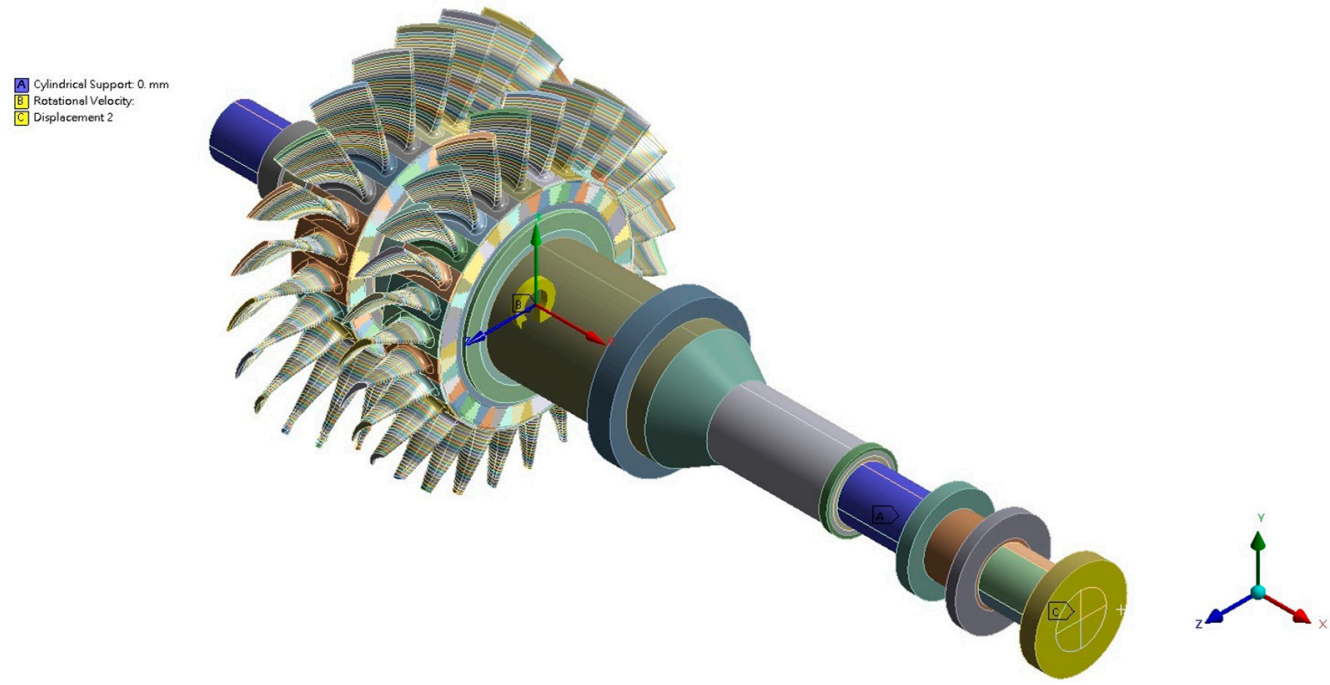

Figure 20. TRT rotor boundary conditions and speed load. 
When the complete loading and boundary are applied to the finite element model, the aerodynamic force analysis is used for evaluating the stress distribution and intensity on the blades, as shown in Figure 21.
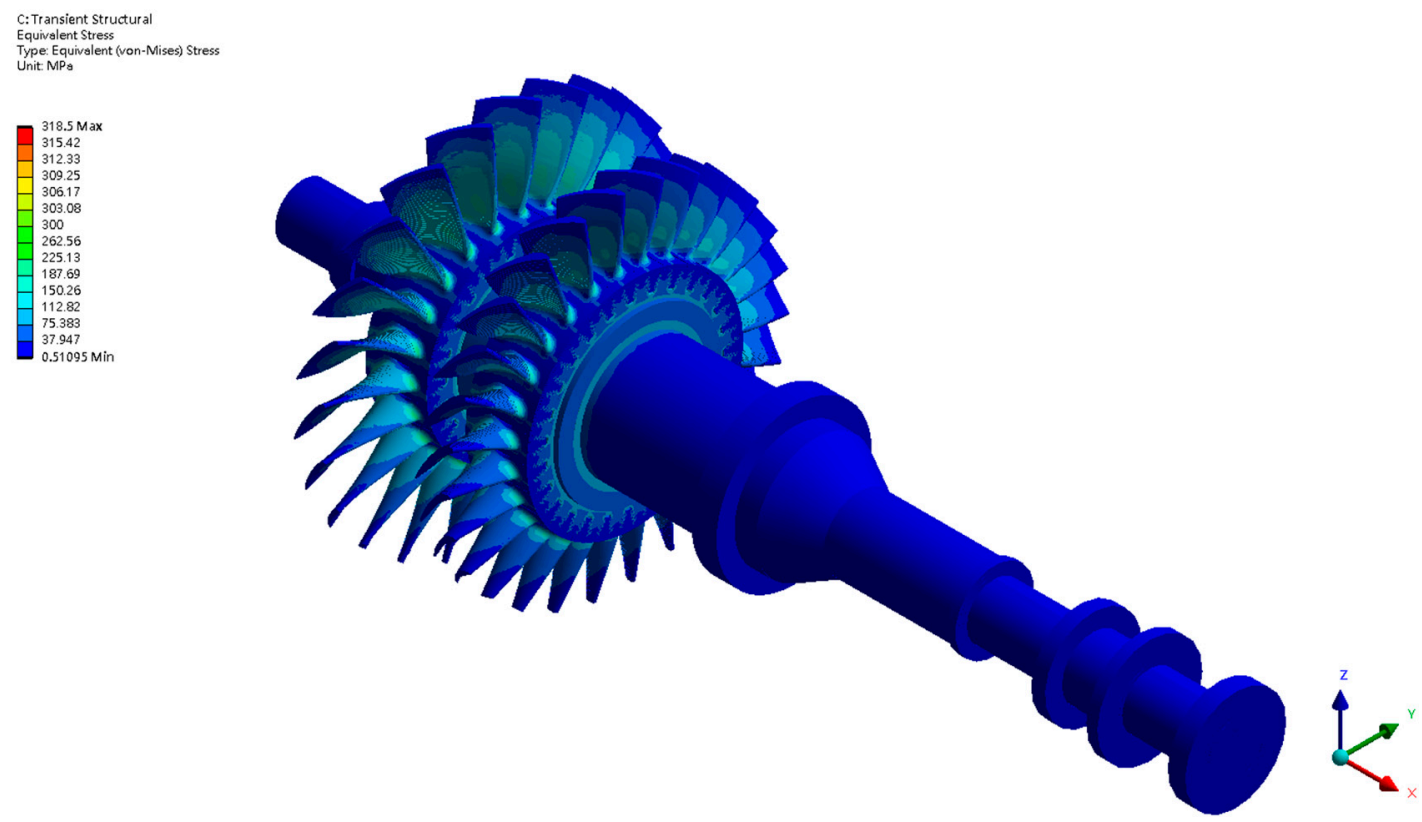

Figure 21. Aerodynamic analysis result of TRT rotor.

According to the analysis results, the position of maximum stress on the TRT rotor in normal working conditions is coincident with the centrifugal force analysis simulation, both in the first section dovetail slot of the blade. According to the simulation results of the 1st stage blade, the stress on the first section dovetail slot of the blade is $282.90 \mathrm{MPa}$, as shown in Figure 22.

C: Transient Structural Equivalent Stress
Type: Equivelent (von-Mises) Stress

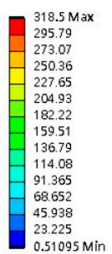

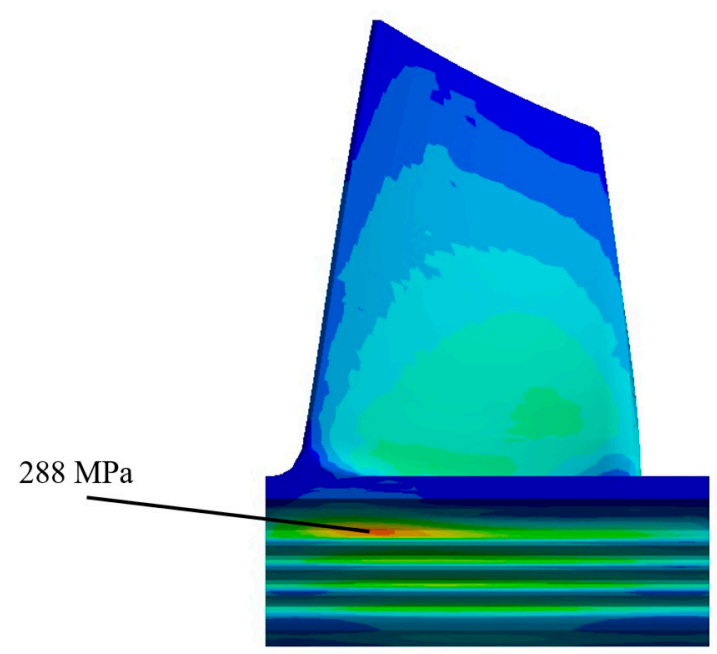

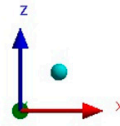

Figure 22. The stress distribution of the 1st stage blade.

The maximum stress on the 2nd stage blade also occurs in the first section dovetail slot of the blade; the stress is $318.50 \mathrm{MPa}$. The stress is the maximum stress on the TRT shaft system, and the stress distribution result is shown in Figure 23. 

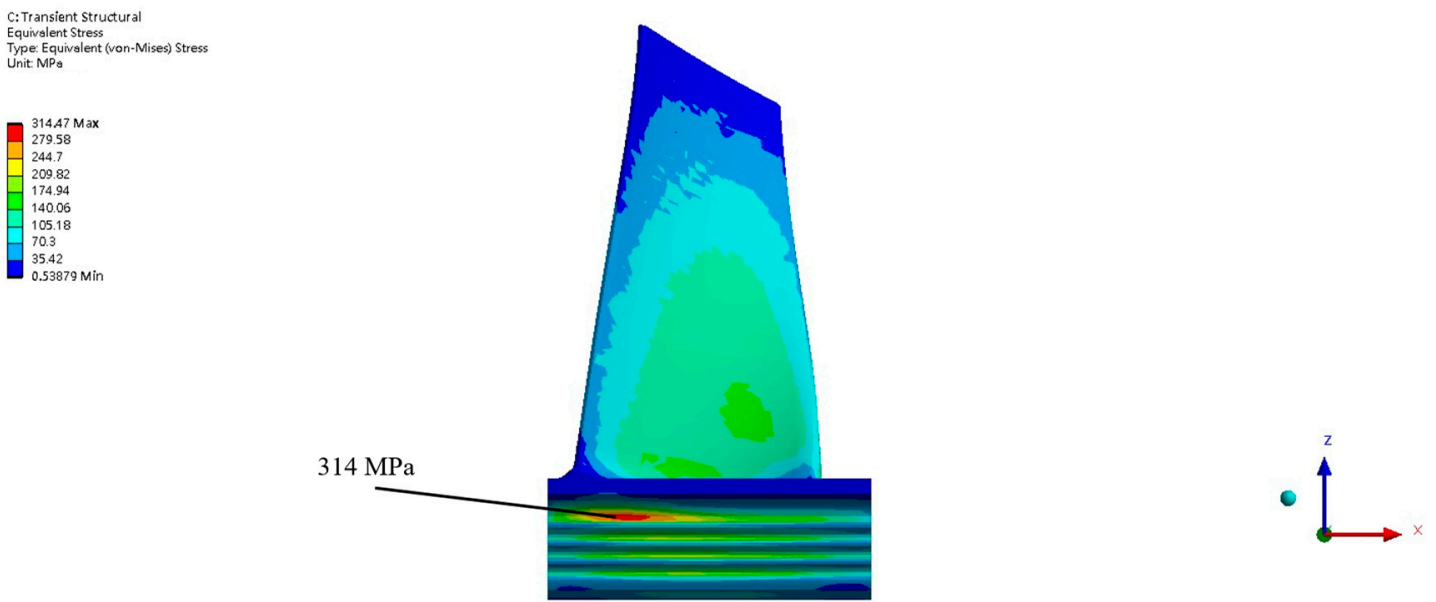

Figure 23. The stress distribution of the 2nd stage blade.

According to the aerodynamic force analysis result, the position of maximum stress on the blade is similar to the position in the centrifugal force result; the maximum stress of the TRT shaft system increases from $286.21 \mathrm{MPa}$ of centrifugal force analysis to $318.50 \mathrm{MPa}$. If the maximum stress is simply used as an evaluation criterion, the TRT structure is stable and safe. As the aerodynamic external force on the TRT rotor in operation is a simple harmonic external force, after long-term usage, the blade structure is likely to have metal fatigue due to aerodynamic external force which may occur in the first section dovetail slot of the blade, as shown in Figure 23.

\subsection{Campbell and SAFE Diagrams of TRT Rotor}

To explore the modal parameters of the TRT rotor when the rotor is running, the modal is excited by the order frequency; the theoretical modal analysis based on the finite element method is employed to explore the dynamic behavior of the rotor at different speeds. The Campbell diagram containing the relationship between the modal frequency and speed of the system at different speeds is employed to evaluate whether the rotating shaft and blades will be excited by the order frequency under the operating speed of the system, which will cause the structural vibration. The SAFE diagram is also applied to discuss and evaluate the structural vibration of the rotor system under the working speed and its corresponding mode shapes.

\subsubsection{Boundary and Load Conditions}

To make the analysis conditions when performing CAE consistent with the real situation, we refer to the engineering drawing of the TRT rotor, impose axial and radial constraints on the bearing, and retain the rotational freedom of the shaft, whose purpose is to simulate the boundary between the bearing and the rotor. The contact surface between the blade and the rotating shaft at the dovetail slot is set to be bonded contact. The reason for this setting is that the interface of the dovetail slot is filled with insulating glue, and there is no relative movement of the interface. In this analysis, the speed range is set from 0 to $3600 \mathrm{rpm}$, and the modal parameters are calculated once at an interval of $600 \mathrm{rpm}$. A total of six sets of modal parameters are obtained. The boundary and loading conditions are shown in Figure 24. 

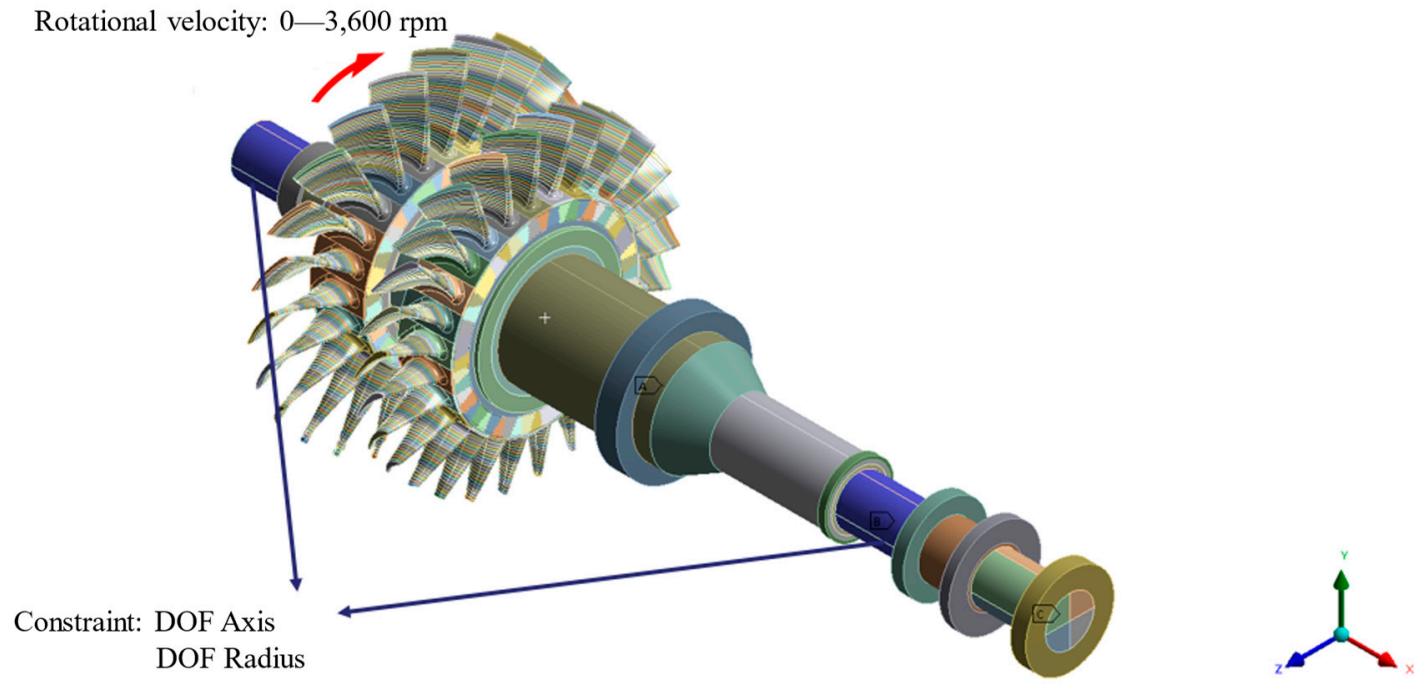

Figure 24. The boundary and loading conditions used in finite element analysis for TRT rotor.

\subsubsection{Analysis Results}

Through the aggregation of the results of modal analysis corresponding to different speeds, the Campbell diagrams of the first- and second-stage moving blades can be obtained, respectively. From the Campbell diagram of the first-stage moving blades, it can be seen that at a working speed of $3600 \mathrm{rpm}$, there are four sets of modes and order lines that overlap. The overlapping order lines are the 22nd, 23rd, 24th, and 27th order; the structural vibration mode may be excited by the first-stage bladed disk at $1630.50 \mathrm{~Hz}, 1504.00 \mathrm{~Hz}$, $1575.10 \mathrm{~Hz}$, and $1543.50 \mathrm{~Hz}$, as shown in Figure 25.

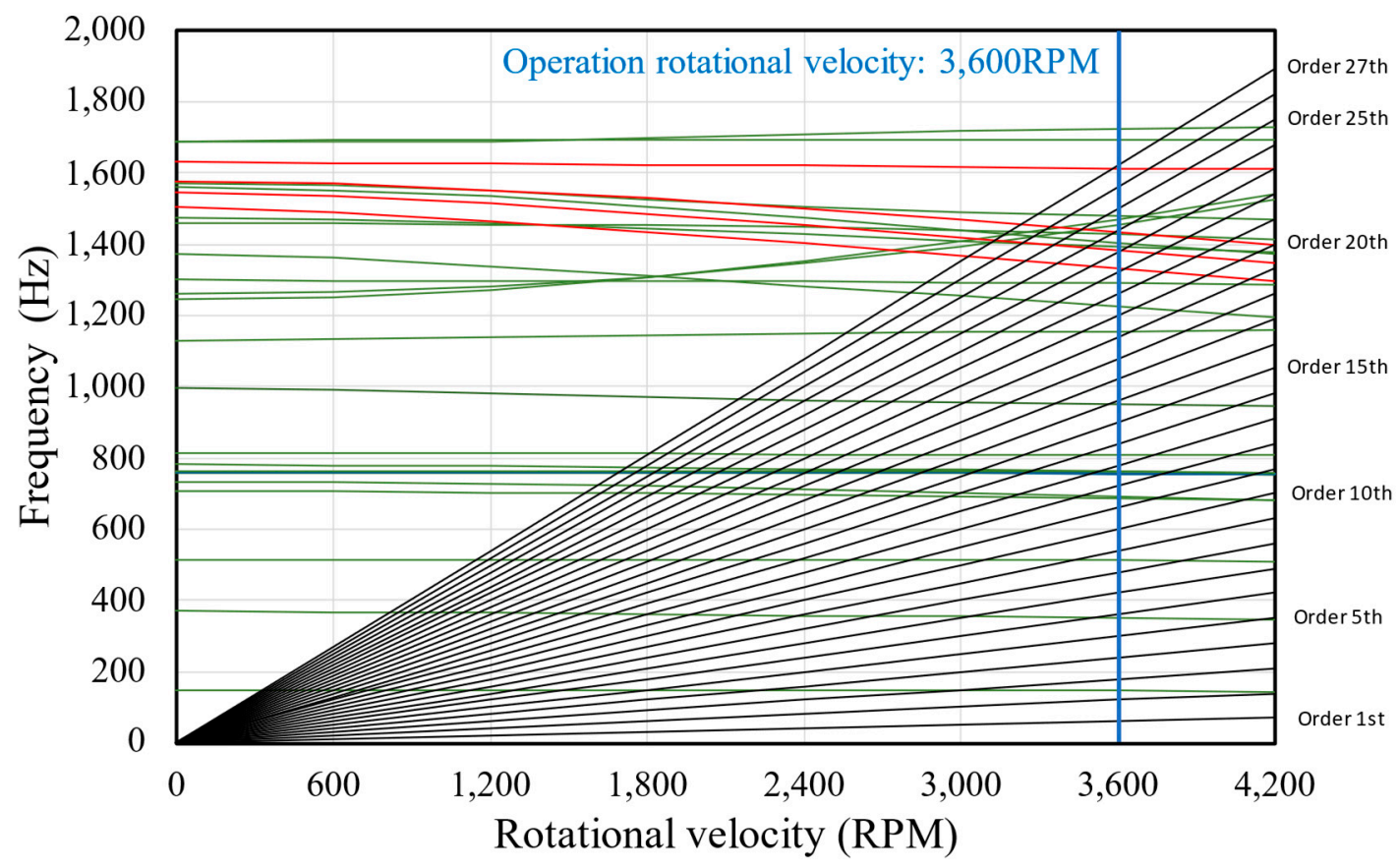

Figure 25. The Campbell diagram of first-stage moving blades.

After obtaining the modal frequencies of the first-stage moving blades and the order line, to explore the types of nodal diameters to corresponding structural modes, we draw the structural modes with different nodal diameters of all the first-stage moving blades at different speeds as SAFE diagrams. After marking the vibration modes with red markers, 
it can be seen that the structural modes are, respectively, 1, 3, and 4 modal diameters, as shown in Figure 26.

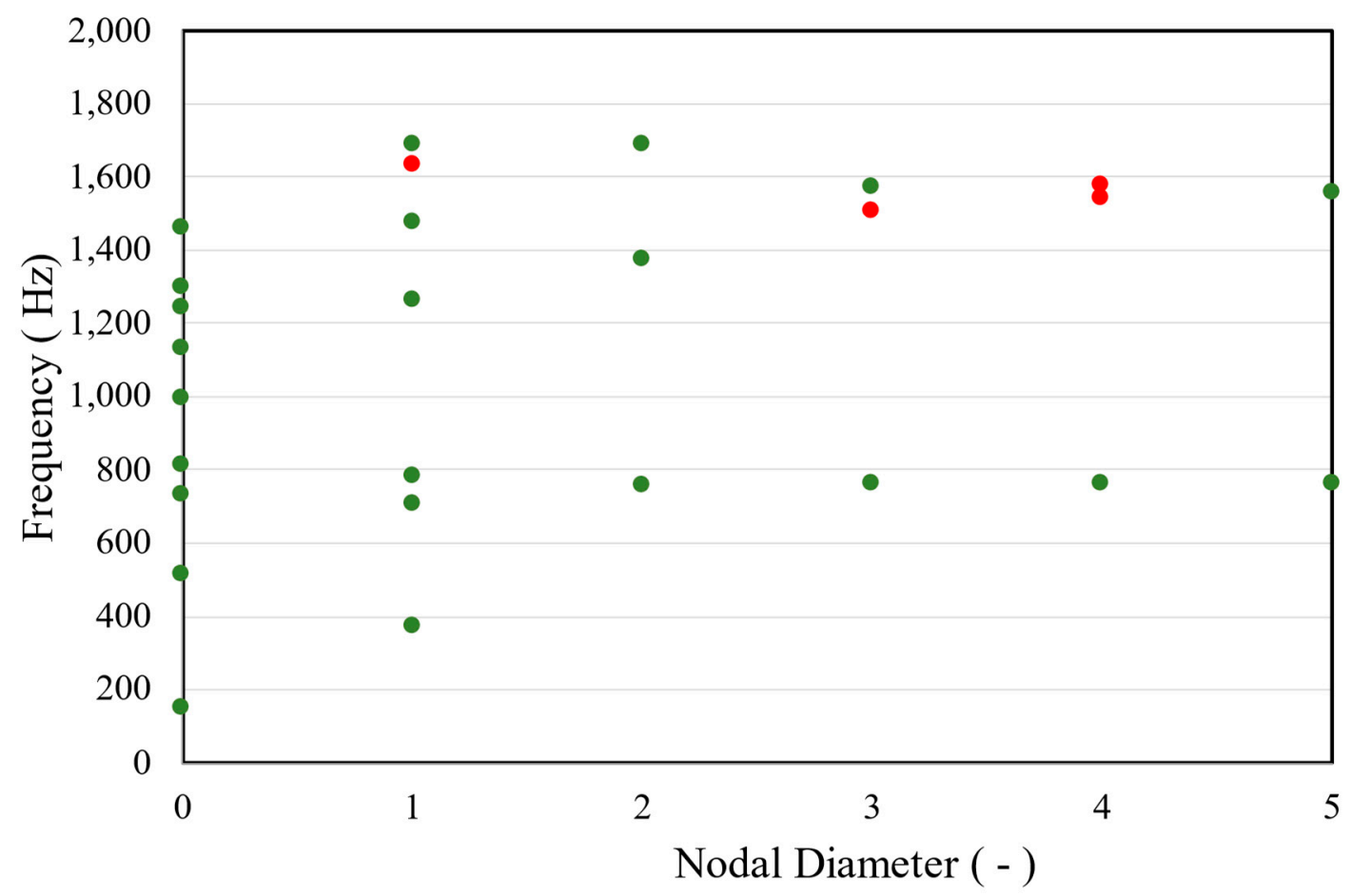

Figure 26. The SAFE diagram of the first-stage moving blade.

It can be seen from the Campbell diagram of the two-stage moving blade that at a working speed of $3600 \mathrm{rpm}$, there are two sets of modes and order lines that overlap. The overlapping order lines are the 7th and 14th orders. The possible structural vibrations of the bladed disk are $438.86 \mathrm{~Hz}$ and $944.43 \mathrm{~Hz}$, as shown in Figure 27.

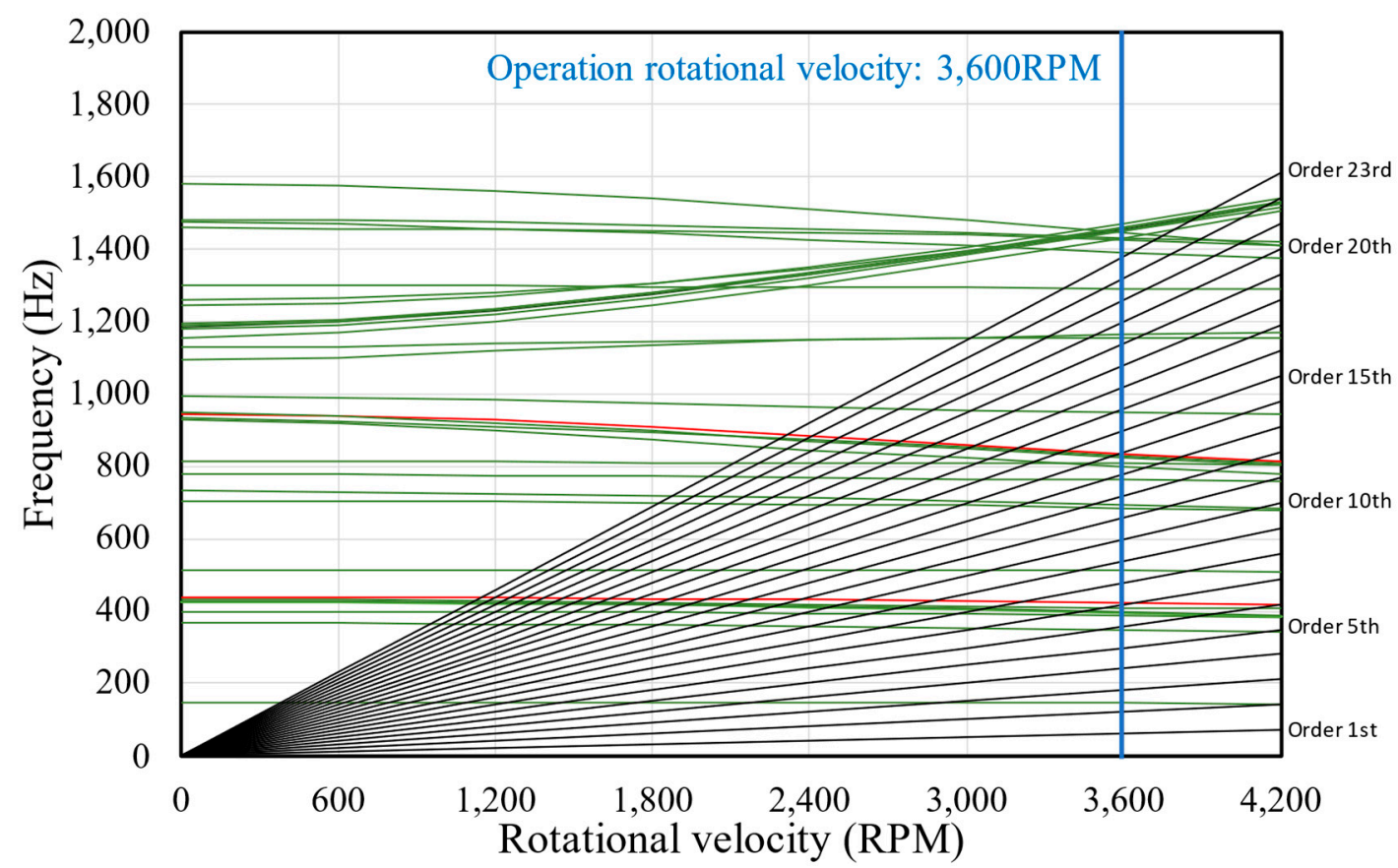

Figure 27. The Campbell diagram of two-stage moving blades. 
After obtaining the modal frequencies of the overlapping of the two-stage moving blades and the order line, to explore the types of nodal diameters to corresponding structural modes, we have drawn the structural modes with different nodal diameters of all the two-stage moving blades at different speeds as SAFE diagrams. After marking the vibration modes with red markers, it can be seen that the respective modes are 1 and 3 modal diameters, as shown in Figure 28.

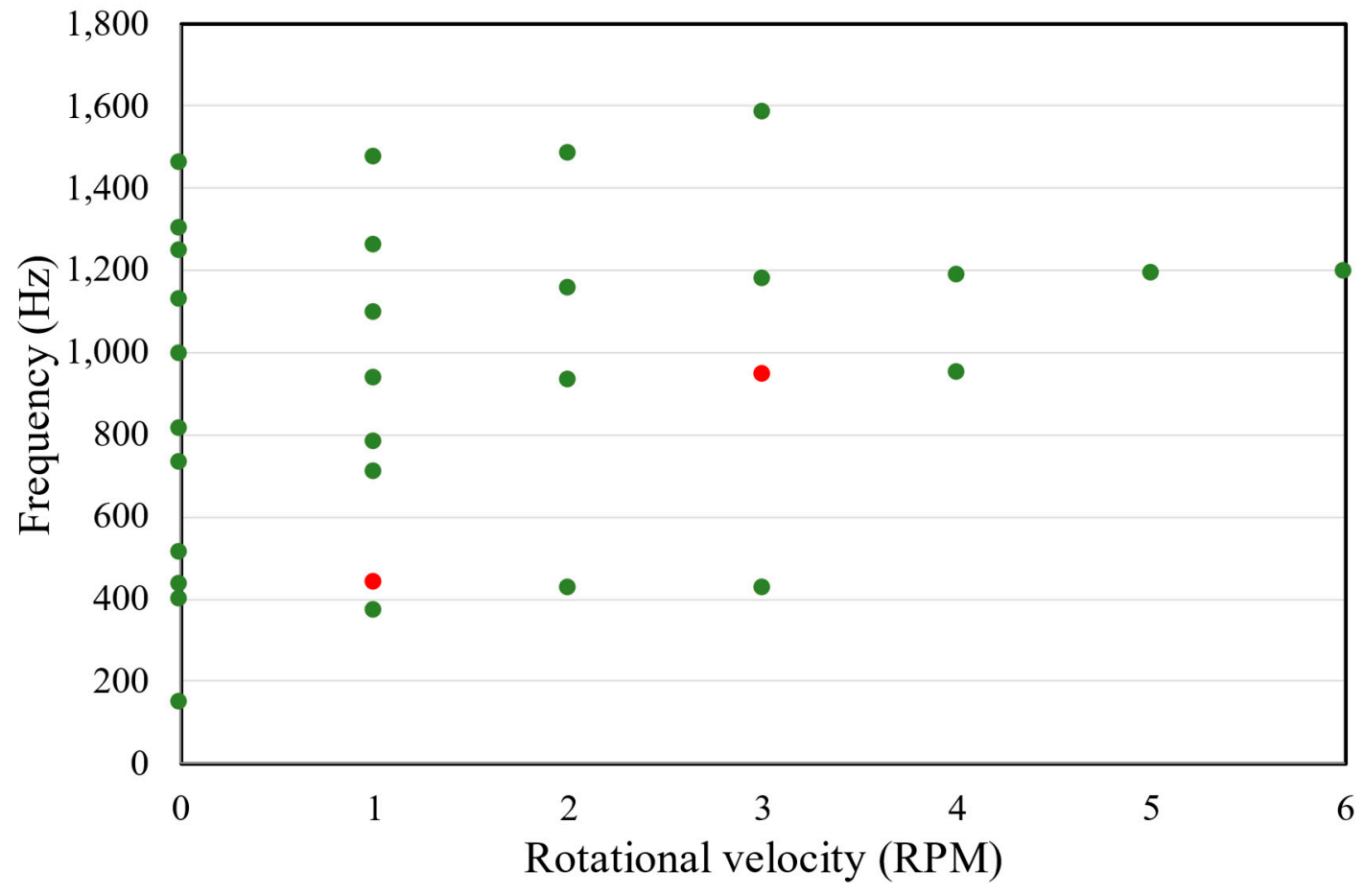

Figure 28. The SAFE diagram of two-stage moving blade.

\section{Conclusions}

This study discussed the aerodynamic force, centrifugal force, and maximum stress on the structure and the positions of occurrence using finite element analysis (FEA) when the first-stage and second-stage moving blades of the TRT rotor system are in rated working conditions. The dynamic characteristics and vibration behaviors of rotor blades are also investigated through Campbell and SAFE diagrams. To validate the effectiveness of the finite element models, the mode shapes and natural frequencies in the FEA-based modal analysis of the TRT rotor are captured and compared with those of the practical structures through experimented modal analysis (EMA). To verify the agreement between the mode shapes of the finite element analysis and those of the actual structure, the modal assurance criterion (MAC) is introduced here to confirm the reliability of the finite element model. We use the modal verification method to explore the reliability of the TRT rotor finite element model. Since the TRT rotor is a large structure and the blade surface attached to the accelerometer is curved, some modal recognition results are poor, but the key modal guarantee indexes of the modal shapes are all higher than 0.6 , so the finite element model is regarded as reliable. The CAE software is used for centrifugal force analysis and aerodynamic analysis to discuss the stress behavior and distribution when the structure is in rated conditions. In centrifugal force analysis, the analytic solution of the average stress of the blade section was compared with simulation results. Their errors are less than $5 \%$, and thus confirm the reliability of simulation from CAE. It is found in the simulation that the first section dovetail slot of the blade bears maximum stress when the rotor blade is in a rated working condition of $3600 \mathrm{rpm}$. The rotor system may have metal fatigue failure in the first section dovetail slot of the blade. To evaluate the possibility of structural vibration of the rotor blades under the working conditions of the TRT rotor structure, we 
gathered the modal parameters at each speed to obtain the Campbell and SAFE diagrams, and determined the modal frequencies and the corresponding modal shapes that may be excited. From the dynamic analysis of the TRT rotor structure, it is pointed out that the key structural resonance frequencies of the primary bladed disk are $1630.50 \mathrm{~Hz}, 1504.00 \mathrm{~Hz}$, $1575.10 \mathrm{~Hz}$, and $1543.50 \mathrm{~Hz}$, all of which are the torsional modal behavior of the blade; the key structural resonance frequency of the secondary blade is $438.86 \mathrm{~Hz}$ and $944.43 \mathrm{~Hz}$, which are the bending and torsion modes of the blade, respectively.

Author Contributions: C.-S.L. conceived of the proposed idea, designed the experiments and verified the data along with H.-T.C., C.-H.H., M.-H.L., J.-K.L. and C.-J.B. The draft preparation is originally written by the first and corresponding author C.-S.L. The article is mainly revised by C.-S.L., reviewed and edited in part by H.-T.C., C.-H.H. and C.-J.B. All authors have read and agreed to the published version of the manuscript.

Funding: This research was supported by China Steel Corporation (CSC) under the Grant RE109704.

Institutional Review Board Statement: Not applicable.

Informed Consent Statement: Not applicable.

Data Availability Statement: Not applicable.

Acknowledgments: This work was supported by Green Energy \& System Integration Research \& Development Department, Technology Division, China Steel Corporation (CSC). The authors would like to thank anonymous reviewers for their valuable comments and suggestions in revising the paper.

Conflicts of Interest: The authors declare no conflict of interest.

\section{References}

1. Wang, R.Q.; Jiang, L.; Wang, Y.D.; Roskilly, A.P. Energy Saving Technologies and Mass-thermal Network Optimization for Decarbonized Iron and Steel Industry: A Review. J. Clean. Prod. 2020, 274, 122997. [CrossRef]

2. Zhang, Q.; Zhao, X.; Lu, H.; Ni, T.; Li, Y. Waste Energy Recovery and Energy Efficiency Improvement in China's Iron and Steel Industry. Appl. Energy 2017, 191, 502-520. [CrossRef]

3. Zhang, M.; Liu, Y.; Wang, W.; Wang, P.; Li, J. The Fatigue of Impellers and Blades. Eng. Fail. Anal. 2016, 62, 208-231. [CrossRef]

4. Wei, Y.; Li, Y.; Lai, J.; Zhao, Q.; Yang, L.; Lin, Q.; Wang, X.; Pan, Z.; Lin, Z. Analysis on Corrosion Fatigue Cracking Mechanism of 17-4PH Blade of Low Pressure Rotor of Steam Turbine. Eng. Fail. Anal. 2020, 118, 104925. [CrossRef]

5. Poursaeidi, E.; Aieneravaie, M.; Mohammadi, M.R. Failure Analysis of a 2nd stage Blade in a Gas Turbine Engine. Eng. Fail. Anal. 2008, 15, 1111-1129. [CrossRef]

6. Sheykhlar, A.B.; Moghanaki, S.K.; Moattari, M.; Shafiei, A.; Amirjan, M. On the Failure Behavior of Fifth Stage Gas Turbine Blade. Eng. Fail. Anal. 2020, 116, 104766. [CrossRef]

7. Katinić, M.; Kozak, D.; Gelo, I.; Damjanović, D. Corrosion Fatigue Failure of Steam Turbine Moving Blades: A Case Study. Eng. Fail. Anal. 2019, 106, 104136. [CrossRef]

8. Zhao, W.; Li, Y.; Xue, M.; Wang, P.; Jiang, J. Vibration Analysis for Failure Detection in Low Pressure Steam Turbine Blades in Nuclear Power Plant. Eng. Fail. Anal. 2018, 84, 11-24. [CrossRef]

9. Liu, M.; Ma, F.; Chang, G.; Fu, F.; Cheruvu, N.S.; Yu, L.; Dai, J.; Xu, K. Experimental Investigation of Failure Behavior of the Cracked 17-4PH Steel Blades in a Top Gas Energy Recovery Turbine. Eng. Fail. Anal. 2019, 105, 545-554. [CrossRef]

10. Cano, S.; Rodríguez, J.A.; Rodríguez, J.M.; García, J.C.; Sierra, F.Z.; Casolco, S.R.; Herreraa, M. Detection of Damage in Steam Turbine Blades Caused by Low Cycle and Strain Cycling Fatigue. Eng. Fail. Anal. 2019, 97, 579-588. [CrossRef]

11. Rodríguez, J.A.; Castro, L.; Tejeda, A.L.; Garcia, J.C.; Rodriguez, J.M.; Galindo, E.; el Hamzaoui, Y. Fatigue of Steam Turbine Blades at Resonance Conditions. Eng. Fail. Anal. 2019, 104, 39-46. [CrossRef]

12. Zhang, M.; Valentin, D.; Valero, C.; Presas, A.; Egusquiza, M.; Egusquiza, E. Experimental and numerical investigation on the influence of a large crack on the modal behaviour of a Kaplan turbine blade. Eng. Fail. Anal. 2020, 109, 104389. [CrossRef]

13. Wu, C.Y. Investigation of a Rotor Blade Dynamics Using Experimental Modal Testing. Technol. Train. 2015, 40, 13-26.

14. Singh, M.P.; Vargo, J.J.; Schiffer, D.M.; Dello, J.D. SAFE Diagram-A Design Reliability Tool for Turbine Blading. In Proceedings of the Seventeenth Turbomachinery Symposium; Turbomachinery Laboratory, Texas A\&M University: College Station, TX, USA, 1988; p. 93. 
15. Bertini, L.; Neri, P.; Santus, C.; Guglielmo, A.; Mariotti, G. Analytical Investigation of the SAFE Diagram for Bladed Wheels, Numerical and Experimental Validation. J. Sound Vib. 2014, 333, 4771-4788. [CrossRef]

16. Madhavan, S.; Jain, R.; Sujatha, C.; Sekhar, A.S. Vibration Based Damage Detection of Rotor Blades in a Gas Turbine Engine. Eng. Fail. Anal. 2014, 46, 26-39. [CrossRef]

17. Allemang, R.J. The Modal Assurance Criterion-Twenty Years of Use and Abuse. Sound Vib. 2003, 37, $14-23$. 